\title{
Water destruction by X-rays in young stellar objects
}

\author{
P. Stäuber ${ }^{1}$, J. K. Jørgensen ${ }^{2}$, E. F. van Dishoeck ${ }^{3}$, S. D. Doty ${ }^{4}$, and A. O. Benz ${ }^{1}$ \\ 1 Institute of Astronomy, ETH Zürich, 8092 Zürich, Switzerland \\ e-mail: pascalst@astro.phys.ethz.ch \\ 2 Harvard-Smithsonian Center for Astrophysics, 60 Garden Street, Cambridge, MA 02138, USA \\ 3 Sterrewacht Leiden, PO Box 9513, 2300 RA Leiden, The Netherlands \\ 4 Department of Physics and Astronomy, Denison University, Granville, OH 43023, USA \\ Received 28 September 2005 / Accepted 27 January 2006
}

ABSTRACT

\begin{abstract}
Aims. We study the $\mathrm{H}_{2} \mathrm{O}$ chemistry in star-forming environments under the influence of a central X-ray source and a central far ultraviolet (FUV) radiation field. The X-ray models are applied to envelopes around low-mass Class 0 and I young stellar objects (YSOs).

Methods. The gas-phase water chemistry is modeled as a function of time, hydrogen density and X-ray flux. To cover a wide range of physical environments, densities between $n_{\mathrm{H}}=10^{4}-10^{9} \mathrm{~cm}^{-3}$ and temperatures between $T=10-1000 \mathrm{~K}$ are studied.

Results. Three different regimes are found: for $T<100 \mathrm{~K}$, the water abundance is of order $10^{-7}-10^{-6}$ and can be somewhat enhanced or reduced due to X-rays, depending on time and density. For $100 \mathrm{~K} \lesssim T \lesssim 250 \mathrm{~K}_{2} \mathrm{H}_{2} \mathrm{O}$ is reduced from initial $x\left(\mathrm{H}_{2} \mathrm{O}\right) \approx 10^{-4}$ following ice evaporation to $x\left(\mathrm{H}_{2} \mathrm{O}\right) \approx 10^{-6}$ for $F_{\mathrm{X}} \gtrsim 10^{-3} \mathrm{erg} \mathrm{s}^{-1} \mathrm{~cm}^{-2}\left(t=10^{4} \mathrm{yr}\right)$ and for $F_{\mathrm{X}} \gtrsim 10^{-4} \mathrm{erg} \mathrm{s}^{-1} \mathrm{~cm}^{-2}\left(t=10^{5} \mathrm{yr}\right)$. At higher temperatures $(T \gtrsim 250 \mathrm{~K})$ and hydrogen densities, water can persist with $x\left(\mathrm{H}_{2} \mathrm{O}\right) \approx 10^{-4}$ even for high $\mathrm{X}$-ray fluxes. Water is destroyed in both Class 0 and I envelopes on relatively short timescales $(t \approx 5000 \mathrm{yr})$ for realistic X-ray fluxes, although the effect is less prominent in Class 0 envelopes due to the higher X-ray absorbing densities there. FUV photons from the central source are not effective in destroying water.

Conclusions. X-rays reduce the water abundances especially in regions where the gas temperature is $T \lesssim 250-300 \mathrm{~K}$ for fluxes $F_{\mathrm{X}} \gtrsim 10^{-5}-10^{-4} \mathrm{erg} \mathrm{s}^{-1} \mathrm{~cm}^{-2}$. The affected regions can be envelopes, disks or outflow hot spots. The average water abundance in Class I sources for $L_{\mathrm{X}} \gtrsim 10^{27} \mathrm{erg} \mathrm{s}^{-1}$ is predicted to be $x\left(\mathrm{H}_{2} \mathrm{O}\right) \lesssim 10^{-6}$. Central UV fields have a negligible influence, unless the photons can escape through cavities.
\end{abstract}

Key words. stars: formation - ISM: molecules - X-rays: ISM - astrochemistry

\section{Introduction}

The importance of water in star-formation is unquestioned. Water is found abundantly on dust grains as well as in the gasphase of molecular clouds, cores, envelopes and protostellar disks. It is a key molecule in the hot $(T \gtrsim 100 \mathrm{~K})$ gas-phase chemistry and is one of the main gas coolants. The evolution over time of molecular cores influences the abundance of $\mathrm{H}_{2} \mathrm{O}$ (Ceccarelli et al. 1996), making it sensitive to the chemical age of a source. Through its masing ability, water has been used to study dynamical properties of star-forming regions.

In cold gas $(T<100 \mathrm{~K})$, water is mainly produced by ionmolecule reactions. At temperatures above $250 \mathrm{~K}, \mathrm{H}_{2} \mathrm{O}$ is largely produced in reactions of $\mathrm{O}$ and $\mathrm{OH}$ with $\mathrm{H}_{2}$. Water is also efficiently generated on dust grains where it forms an icy mantle. Observed abundances of water ice are typically $x\left(\mathrm{H}_{2} \mathrm{O}\right) \approx 10^{-4}$ with respect to $\mathrm{H}_{2}$ (Tielens et al. 1991; Boogert et al. 2004). At temperatures $T \gtrsim 100 \mathrm{~K}, \mathrm{H}_{2} \mathrm{O}$ evaporates into the gas-phase and has a major impact on the chemistry (van Dishoeck \& Blake 1998; Doty et al. 2002). Water is also believed to be formed in shocked regions where it may be produced due to the high gas temperature and/or may be released from grains. Towards high-mass star-forming regions, hot $(T \gtrsim 100 \mathrm{~K})$ gas-phase water is usually observed with abundances $x\left(\mathrm{H}_{2} \mathrm{O}\right)=10^{-4}$, comparable to the ice abundance - whereas at temperatures below $100 \mathrm{~K}$, it is found to be approximately 100 times less abundant (e.g., Boonman \& van Dishoeck 2003; van der Tak et al. 2006).
The observed water emission towards low-mass objects has been interpreted with similar jumps at $T \approx 100 \mathrm{~K}$ (Ceccarelli et al. 2000; Maret et al. 2002). Although the solid water abundances are comparable in low and high-mass regions, the hot gas-phase water in low-mass sources seems to be much less abundant than in the massive objects. In addition, a fraction of the emission is believed to arise in outflow regions, as inferred from observations both on and off source with the Long Wavelength Spectrometer (LWS) on board the Infrared Space Observatory (ISO) (Giannini et al. 2001; Benedettini et al. 2002). Nisini et al. (2002) compared the far-infrared spectra of both Class 0 and Class I sources. Water lines were found to be prominent in the spectra of Class 0 objects but they were not detected toward Class I sources corresponding to an upper limit on the abundances of $x\left(\mathrm{H}_{2} \mathrm{O}\right) \lesssim 10^{-5}$.

Protostars are often found to be sources of strong X-ray fluxes (e.g., Casanova et al. 1995; Koyama et al. 1996; Imanishi et al. 2001). The observed X-rays are understood to be thermal emission from magnetic stellar activities or from the disk-star system (e.g., Feigelson \& Montmerle 1999). Typical $\mathrm{X}$-ray luminosities range from $L_{\mathrm{X}} \approx 10^{28}-10^{31} \mathrm{erg} \mathrm{s}^{-1}$ in the $0.5-10 \mathrm{keV}$ band with plasma temperatures between $0.6-7 \mathrm{keV}$ (e.g., Imanishi et al. 2001). Flares can even lead to plasma temperatures temporarily exceeding $10^{8} \mathrm{~K}$ with luminosities higher than $L_{\mathrm{X}} \approx 10^{32} \mathrm{erg} \mathrm{s}^{-1}$. Within the Chandra Orion Ultradeep Project (COUP), Preibisch et al. (2005) found a ratio for the 
X-ray luminosity to the bolometric luminosity of $\log \left(L_{\mathrm{X}} / L_{\mathrm{bol}}\right) \approx$ -3.6 for Class I and older protostars. No X-ray detection has been reported to date toward Class 0 objects (Hamaguchi et al. 2005a). However, this might be due to the large X-ray absorbing hydrogen and dust column densities found in these sources. The nature of the sources in recent reports of X-ray detections towards very young objects is poorly known and they could be either Class 0 or I objects (Hamaguchi et al. 2005b; Forbrich et al. 2005).

The influence of a central X-ray source on the envelope around young stellar objects has recently been studied by Stäuber et al. (2005). It was found that the ionization rate in the inner region of the surrounding envelope may be dominated by the ionizing X-ray flux rather than by an inner far ultraviolet (FUV) radiation field or the cosmic-ray ionization rate. The ionizing flux enhances $\mathrm{H}_{3}^{+}$and $\mathrm{He}^{+}$that trigger a distinct chemistry. It was also found that the $\mathrm{H}_{2} \mathrm{O}$ abundances in the gas-phase may be reduced due to X-rays. The abundance and behavior of $\mathrm{H}_{2} \mathrm{O}$ in both the solid and the gas-phase will be studied extensively with the upcoming Herschel Space Observatory. It is therefore timely to investigate the influence of X-rays and inner FUV fields on the abundances of gas-phase water in more detail.

In this paper, we first study the general influence of X-rays on gas-phase water. The time-dependent chemical X-ray models of Stäuber et al. (2005) are used and applied to different density and temperature regimes for different X-ray fluxes (Sect. 2). The densities and temperatures are chosen to cover a wide range of conditions that are applicable to cold molecular clouds, envelopes, protostellar disks and outflow hot spots. Regions with density and temperature gradients are modeled and discussed in Sect. 3. These regions are representative of low-mass Class 0 and I envelopes. In addition to X-rays, central FUV fields are considered. The aim is to find systematic trends between the different type of sources. The results for the different envelopes are discussed in Sect. 4. The conclusions are drawn in Sect. 5.

\section{General parameter study}

The gas-phase water abundance is modeled as a function of time, hydrogen density $\left(n_{\mathrm{H}}=n(\mathrm{H})+2 n\left(\mathrm{H}_{2}\right)\right)$ and X-ray flux. The results of this study can be applied to a wide range of different physical environments including protostellar envelopes and disks.

\subsection{Chemical model}

The X-ray chemistry models are described in detail by Stäuber et al. (2005). The models are an extension of the gas-phase chemical models of Doty et al. $(2002,2004)$ to allow the impact of X-rays from the central source on the surrounding molecular envelope. The input parameters for the model are the initial molecular or atomic abundances, the hydrogen density, the gas temperature, the X-ray luminosity, the X-ray emitting plasma temperature, the X-ray absorbing hydrogen column density, the cosmic-ray ionization rate and the enhancement of the inner and outer FUV field with respect to the average interstellar radiation field (ISRF).

The chemical model is based on the UMIST gas-phase chemical reaction network (Millar et al. 1997) and calculates the time-dependent number density $n($ i) of each species for a certain temperature and distance from the source. For the initial chemical abundances we follow the models of Doty et al. $(2002,2004)$ for the high-mass source AFGL 2591 and low-mass source
Table 1. Initial abundances and cosmic-ray ionization rate.

\begin{tabular}{|c|c|c|}
\hline Species & Initial abundance & Ref \\
\hline \multicolumn{3}{|l|}{ Initial abundances $T>100 \mathrm{~K}$} \\
\hline $\mathrm{CO}$ & $2.0 \mathrm{E}-04$ & a \\
\hline $\mathrm{CO}_{2}$ & $3.0 \mathrm{E}-05$ & $\mathrm{~b}$ \\
\hline $\mathrm{H}_{2} \mathrm{O}$ & $1.5 \mathrm{E}-04$ & $\mathrm{c}$ \\
\hline $\mathrm{H}_{2} \mathrm{~S}$ & $1.0 \mathrm{E}-08$ & $\mathrm{~d}$ \\
\hline $\mathrm{H}_{2} \mathrm{CO}$ & $8.0 \mathrm{E}-08$ & $\mathrm{~d}$ \\
\hline $\mathrm{N}_{2}$ & 7.0E-05 & e \\
\hline $\mathrm{CH}_{4}$ & $1.0 \mathrm{E}-07$ & $\mathrm{e}$ \\
\hline $\mathrm{C}_{2} \mathrm{H}_{4}$ & $8.0 \mathrm{E}-08$ & e \\
\hline $\mathrm{C}_{2} \mathrm{H}_{6}$ & $1.0 \mathrm{E}-08$ & e \\
\hline $\mathrm{CH}_{3} \mathrm{OH}$ & $1.5 \mathrm{E}-07$ & $\mathrm{~d}$ \\
\hline $\mathrm{O}$ & 0.0 & $\mathrm{e}$ \\
\hline S & 0.0 & $\mathrm{e}$ \\
\hline \multicolumn{3}{|l|}{ Initial abundances $T<100 \mathrm{~K}$} \\
\hline $\mathrm{CO}$ & $2.0 \mathrm{E}-04$ & $\mathrm{~d}$ \\
\hline $\mathrm{CO}_{2}$ & 0.0 & $\mathrm{f}$ \\
\hline $\mathrm{H}_{2} \mathrm{O}$ & 0.0 & $\mathrm{f}$ \\
\hline $\mathrm{H}_{2} \mathrm{~S}$ & 0.0 & $\mathrm{f}$ \\
\hline $\mathrm{N}_{2}$ & $7.0 \mathrm{E}-05$ & $\mathrm{e}$ \\
\hline $\mathrm{CH}_{4}$ & $1.0 \mathrm{E}-07$ & e \\
\hline $\mathrm{C}_{2} \mathrm{H}_{4}$ & $8.0 \mathrm{E}-08$ & e \\
\hline $\mathrm{C}_{2} \mathrm{H}_{6}$ & $1.0 \mathrm{E}-08$ & $\mathrm{e}$ \\
\hline $\mathrm{H}_{2} \mathrm{CO}(60<T(\mathrm{~K})<100)$ & $8.0 \mathrm{E}-08$ & $\mathrm{~d}$ \\
\hline $\mathrm{H}_{2} \mathrm{CO}(T(\mathrm{~K})<60)$ & 0.0 & $\mathrm{~d}$ \\
\hline $\mathrm{CH}_{3} \mathrm{OH}(60<T(\mathrm{~K})<100)$ & $1.5 \mathrm{E}-07$ & $\mathrm{~d}$ \\
\hline $\mathrm{CH}_{3} \mathrm{OH}(T(\mathrm{~K})<60)$ & 0.0 & $\mathrm{~d}$ \\
\hline $\mathrm{O}$ & $1.0 \mathrm{E}-04$ & $\mathrm{~d}$ \\
\hline S & $6.0 \mathrm{E}-09$ & $\mathrm{~g}$ \\
\hline Cosmic-ray ionization rate $\zeta_{\mathrm{cr}}\left(\mathrm{s}^{-1}\right)$ & $0.8 \mathrm{E}-17$ & $\mathrm{~h}$ \\
\hline
\end{tabular}

All abundances are relative to molecular hydrogen. ${ }^{a}$ Jørgensen et al. (2002), ${ }^{b}$ Boonman et al. (2003a), ${ }^{c}$ Boonman \& van Dishoeck (2003), ${ }^{d}$ Doty et al. (2004), ${ }^{e}$ Charnley (1997), ${ }^{f}$ assumed to be frozen-out or absent in cold gas-phase, ${ }^{g}$ Doty et al. (2002), ${ }^{h}$ see text.

IRAS 16293-2422. These models successfully reproduced many of the observed molecular lines. The effects of evaporation of a certain species into the gas-phase have been approximated by initially depleting this species below its evaporation temperature $T_{\text {ev }}$. Specifically, all $\mathrm{H}_{2} \mathrm{O}$ is assumed to evaporate at $T>100 \mathrm{~K}$. No photodesorption of ice is taken into account. The initial abundances are listed in Table 1. The adopted cosmic-ray ionization rate is discussed in Sect. 3.1.

The gas temperature has been taken to be closely coupled to the dust temperature. Although the X-ray flux and the gas temperature are not fully independent, they have been treated as uncoupled variables in order to study the dependence of $\mathrm{H}_{2} \mathrm{O}$ on each of them. To cover a wide range of different physical environments, the hydrogen density is varied between $n_{\mathrm{H}}=10^{4}-10^{9} \mathrm{~cm}^{-3}$. At higher densities, the mean free path of $\mathrm{X}$-rays becomes small (a few AU). Typical densities in the inner part of envelopes or protoplanetary disk atmospheres are between $n_{\mathrm{H}}=10^{6}-10^{9} \mathrm{~cm}^{-3}$, whereas those for outflows are $n_{\mathrm{H}}=10^{4}-10^{6} \mathrm{~cm}^{-3}$. The adopted gas temperature is between $10-1000 \mathrm{~K}$. At higher temperatures $(T \gtrsim 2000 \mathrm{~K})$, molecular hydrogen and water are dissociated due to $\mathrm{H}$ and $\mathrm{H}_{2}$ collisions. The modeled X-ray fluxes are between $F_{\mathrm{X}}=10^{-6}-10 \mathrm{erg} \mathrm{s}^{-1} \mathrm{~cm}^{-2}$. The initial X-ray spectrum is reduced preferentially at low energies by the intervening hydrogen column density such that the local X-ray flux roughly scales with

$F_{\mathrm{X}} \approx\left(\frac{L_{\mathrm{X}}}{10^{31} \mathrm{erg} \mathrm{s}^{-1}}\right)\left(\frac{N_{\mathrm{H}}}{5.0 \times 10^{21} \mathrm{~cm}^{-2}}\right)^{-\gamma}\left(\frac{r}{56 \mathrm{AU}}\right)^{-2}$ 

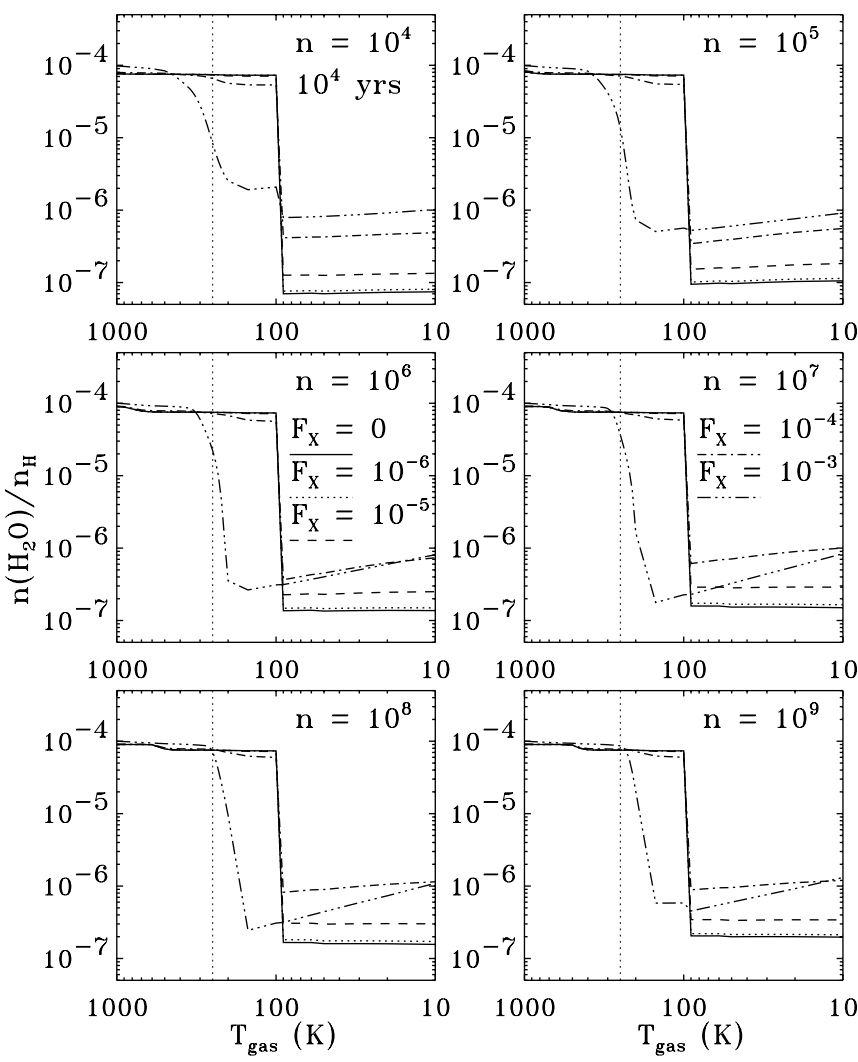

Fig. 1. Fractional water abundances as functions of the gas temperature and low X-ray flux $\left(F_{\mathrm{X}}=10^{-6}-10^{-3} \mathrm{erg} \mathrm{s}^{-1} \mathrm{~cm}^{-2}\right)$ for different densities $\left(\mathrm{cm}^{-3}\right)$ at $t=10^{4} \mathrm{yr}$. The vertical line indicates the $250 \mathrm{~K}$ temperature mark.

in $\mathrm{erg} \mathrm{s}^{-1} \mathrm{~cm}^{-2}$, where $L_{\mathrm{X}}$ is the original X-ray luminosity, $N_{\mathrm{H}}$ the X-ray absorbing column density and $r$ is the distance from the source. The X-ray fluxes are accurate within $20 \%$ for $T_{\mathrm{X}}=$ $3 \times 10^{7} \mathrm{~K}$ and $N_{\mathrm{H}}=5 \times 10^{21}-10^{23} \mathrm{~cm}^{-2}$ with $\gamma=0.3$, for $N_{\mathrm{H}}=2-$ $5 \times 10^{23} \mathrm{~cm}^{-2}$ with $\gamma=0.4$ and for $N_{\mathrm{H}}=6 \times 10^{23}-10^{24} \mathrm{~cm}^{-2}$ with $\gamma=0.5$. A column density of $N_{\mathrm{H}}=5 \times 10^{21} \mathrm{~cm}^{-2}$ absorbs all photons below $\approx 1 \mathrm{keV}$ (Stäuber et al. 2005). The X-ray spectrum is assumed to be thermal with a plasma temperature $T_{\mathrm{X}}=3 \times 10^{7} \mathrm{~K}(2.6 \mathrm{keV})$. The shape of the X-ray spectrum, however, has only little influence on the chemistry (Maloney et al. 1996; Stäuber et al. 2005). FUV fields are neglected.

All reaction rates that are relevant for the $\mathrm{H}_{2} \mathrm{O}$ chemistry (Sect. 2.3) are given with a maximum uncertainty of $25 \%$ by the UMIST database ${ }^{1}$. Self-shielding of $\mathrm{CO}$ has been included by using the shielding functions of Lee et al. (1996).

\subsection{Results}

The model results for different $\mathrm{X}$-ray fluxes and hydrogen densities are shown in Figs. 1 and 2 for $t=10^{4} \mathrm{yr}$ and in Figs. 3 and 4 for $t=10^{5} \mathrm{yr}$. It can be seen in Figs. 1 and 3 that X-ray fluxes $F_{\mathrm{X}} \lesssim 10^{-6} \mathrm{erg} \mathrm{s}^{-1} \mathrm{~cm}^{-2}$ have no or minor influence on the water abundances for the densities considered. For higher X-ray fluxes, three characteristic regimes can be distinguished: 1 . the regime at $T<100 \mathrm{~K}$, where gas-phase water is mainly formed and destroyed in ion-molecule reactions $\left(x\left(\mathrm{H}_{2} \mathrm{O}\right) \approx 10^{-7}-10^{-6}\right), 2$. the regime $100 \mathrm{~K} \lesssim T \lesssim 250 \mathrm{~K}$, where water is released from grains, but destroyed by $\mathrm{X}$-rays $\left(x\left(\mathrm{H}_{2} \mathrm{O}\right) \approx 10^{-6}\right)$ and 3 . The regime $T \gtrsim 250 \mathrm{~K}$, where the water abundance is $x\left(\mathrm{H}_{2} \mathrm{O}\right) \gtrsim 10^{-4}$.

\footnotetext{
${ }^{1}$ http://www.rate99.co.uk
}
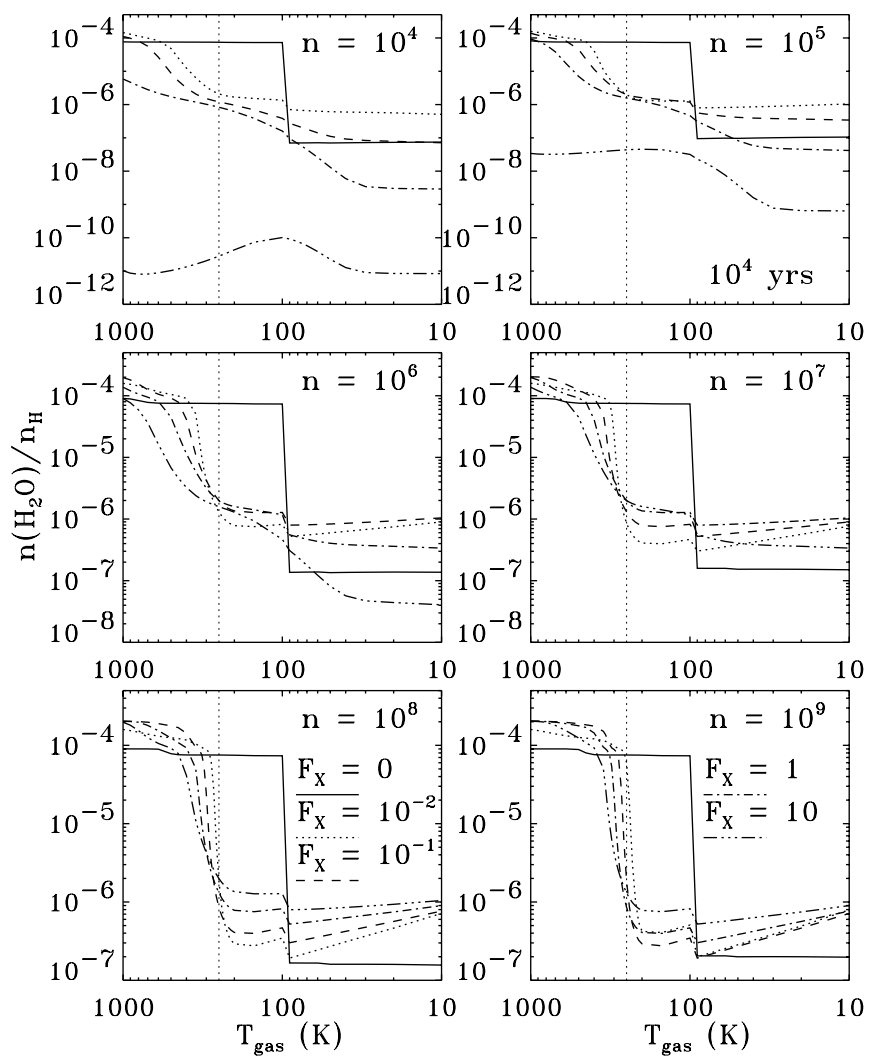

Fig. 2. Fractional water abundances as functions of the gas temperature and high X-ray flux $\left(F_{\mathrm{X}}=10^{-2}-10 \mathrm{erg} \mathrm{s}^{-1} \mathrm{~cm}^{-2}\right)$ for different densities $\left(\mathrm{cm}^{-3}\right)$ at $t=10^{4} \mathrm{yr}$. The vertical line indicates the $250 \mathrm{~K}$ temperature mark.
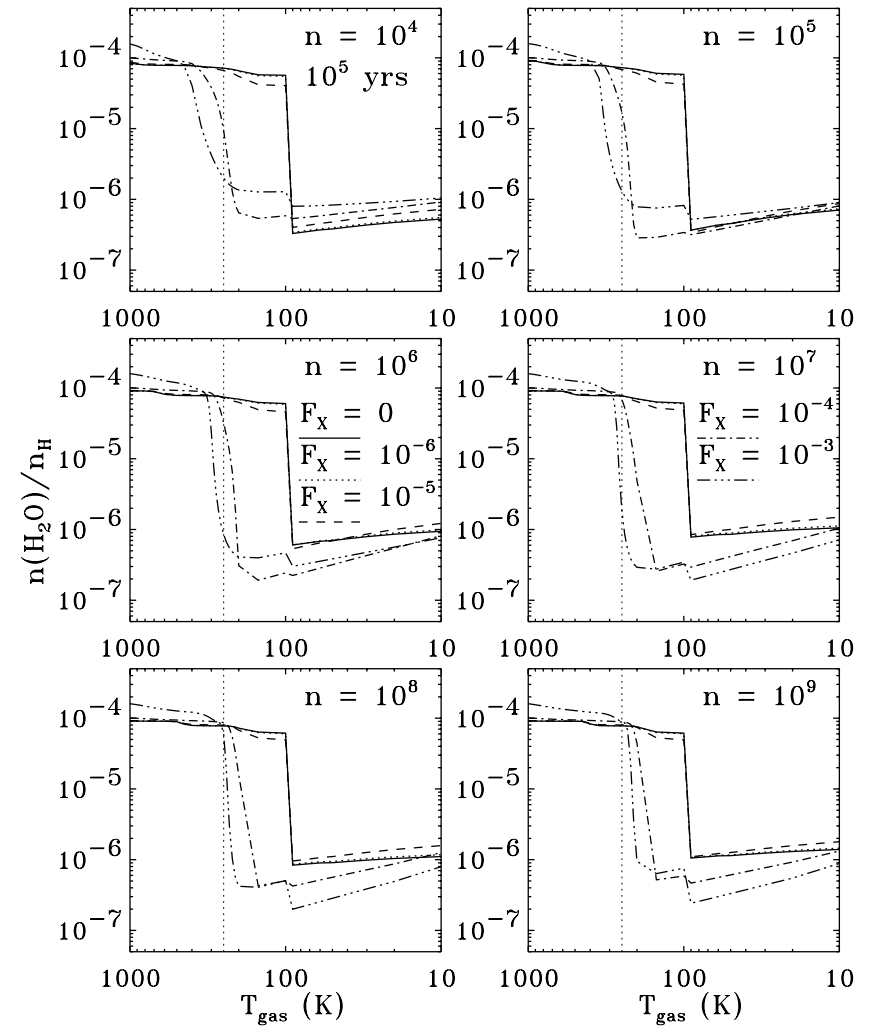

Fig. 3. Fractional water abundances as functions of the gas temperature and low X-ray flux $\left(F_{\mathrm{X}}=10^{-6}-10^{-3} \mathrm{erg} \mathrm{s}^{-1} \mathrm{~cm}^{-2}\right)$ for different densities $\left(\mathrm{cm}^{-3}\right)$ at $t=10^{5} \mathrm{yr}$. The vertical line indicates the $250 \mathrm{~K}$ temperature mark. 

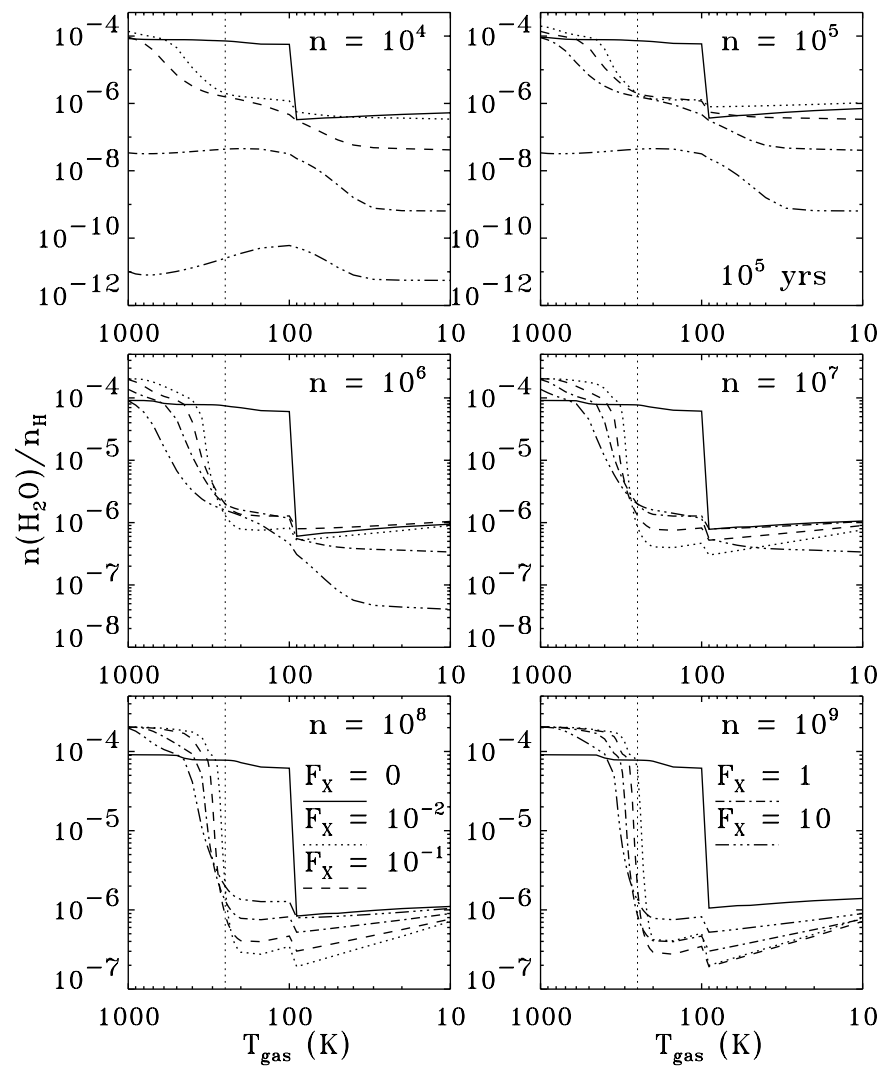

Fig. 4. Fractional water abundances as functions of the gas temperature and high X-ray flux $\left(F_{\mathrm{X}}=10^{-2}-10 \mathrm{erg} \mathrm{s}^{-1} \mathrm{~cm}^{-2}\right)$ for different densities $\left(\mathrm{cm}^{-3}\right)$ at $t=10^{5} \mathrm{yr}$. The vertical line indicates the $250 \mathrm{~K}$ temperature mark.

The three regimes are discussed in some detail in the following paragraphs.

At $t=10^{4} \mathrm{yr}$, X-ray fluxes $10^{-5} \lesssim F_{\mathrm{X}} \lesssim 10^{-2} \mathrm{erg} \mathrm{s}^{-1} \mathrm{~cm}^{-2}$ enhance the water abundance for temperatures $T<100 \mathrm{~K}$ compared to the model without $\mathrm{X}$-rays. The reason for this is the increased $\mathrm{H}_{3} \mathrm{O}^{+}$abundance due to X-rays. $\mathrm{H}_{3} \mathrm{O}^{+}$can recombine quickly to $\mathrm{H}_{2} \mathrm{O}$ in reactions with electrons. Note that net formation of $\mathrm{H}_{2} \mathrm{O}$ at these fluxes can occur because $\mathrm{H}_{3} \mathrm{O}^{+}$does not require $\mathrm{H}_{2} \mathrm{O}$ in its formation. It is produced by ion-molecule reactions starting from $\mathrm{CO}, \mathrm{CO}_{2}, \mathrm{O}, \mathrm{O}_{2}$ and $\mathrm{OH}$, which exist in the gas phase. Higher X-ray fluxes, however, can enhance water only at densities $n_{\mathrm{H}} \gtrsim 10^{7} \mathrm{~cm}^{-3}$ for $T<100 \mathrm{~K}$ (Fig. 2). At $t=10^{5} \mathrm{yr}$, water is only enhanced at $n_{\mathrm{H}}=10^{4} \mathrm{~cm}^{-3}$ in this temperature regime (Fig. 3). At higher densities, water is less abundant in models with X-rays compared to those without X-rays.

For $100 \mathrm{~K} \lesssim T \lesssim 250 \mathrm{~K}$, the initial abundance of water is much higher due to ice evaporation but this high abundance rapidly decreases for $F_{\mathrm{X}} \gtrsim 10^{-3} \mathrm{erg} \mathrm{s}^{-1} \mathrm{~cm}^{-2}$ at $t=10^{4} \mathrm{yr}$ and for $F_{\mathrm{X}} \gtrsim 10^{-4} \mathrm{erg} \mathrm{s}^{-1} \mathrm{~cm}^{-2}$ at $t=10^{5} \mathrm{yr}$. $\mathrm{H}_{2} \mathrm{O}$ is mainly destroyed in reactions with $\mathrm{H}_{3}^{+}$and $\mathrm{HCO}^{+}$whose abundances are enhanced due to the $\mathrm{X}$-rays. Another destruction mechanism is the dissociation of $\mathrm{H}_{2} \mathrm{O}$ by FUV photons created locally by excited $\mathrm{H}_{2}$. The rate for the X-ray induced FUV destruction of water is calculated with the method and numbers provided by Maloney et al. (1996). It is an adaption of the treatment of Gredel et al. (1989) for the effects of internally generated FUV photons by cosmic rays.

In the temperature regime $T \gtrsim 250 \mathrm{~K}$, the reaction $\mathrm{OH}+$ $\mathrm{H}_{2} \rightarrow \mathrm{H}_{2} \mathrm{O}+\mathrm{H}$ can become more efficient than the water destroying reactions and $\mathrm{H}_{2} \mathrm{O}$ has abundances of the order of $10^{-4}$.
At low densities $\left(n_{\mathrm{H}}=10^{4}-10^{5} \mathrm{~cm}^{-3}\right)$, high X-ray fluxes lead to the destruction of water even for high temperatures. Although the gas temperature at these high fluxes may be more than $1000 \mathrm{~K}$, water does not reach fractional abundances higher than $x\left(\mathrm{H}_{2} \mathrm{O}\right) \approx 10^{-7}$, since $\mathrm{H}_{2} \mathrm{O}$ is destroyed in collisions with $\mathrm{H}$ and $\mathrm{H}_{2}$ at higher temperatures. The water abundance is therefore $x\left(\mathrm{H}_{2} \mathrm{O}\right) \lesssim 10^{-10}$ for $F_{\mathrm{X}}=10 \mathrm{erg} \mathrm{s}^{-1} \mathrm{~cm}^{-2}$ and $n_{\mathrm{H}}=10^{4} \mathrm{~cm}^{-3}$ and $x\left(\mathrm{H}_{2} \mathrm{O}\right) \lesssim 10^{-7}$ for $n_{\mathrm{H}}=10^{5} \mathrm{~cm}^{-3}$, independent of the gas temperature. At higher densities, however, even high X-ray fluxes cannot destroy water in the regime $T \gtrsim 250 \mathrm{~K}$. At densities $n_{\mathrm{H}} \gtrsim 10^{7} \mathrm{~cm}^{-3}$, X-rays even enhance the water abundance $\left(x\left(\mathrm{H}_{2} \mathrm{O}\right)>3 \times 10^{-4}\right)$ for temperatures $T \gtrsim 300-600 \mathrm{~K}$ compared to models without $\mathrm{X}$-rays. This enhancement is again mainly through recombination reactions of $\mathrm{H}_{3} \mathrm{O}^{+}$.

At later times $\left(t=10^{6} \mathrm{yr}\right)$, water is destroyed in the regime $100 \mathrm{~K} \lesssim T \lesssim 250 \mathrm{~K}$ for $F_{\mathrm{X}} \gtrsim 10^{-5} \mathrm{erg} \mathrm{s}^{-1} \mathrm{~cm}^{-2}$. The other temperature regimes in the $\mathrm{X}$-ray models resemble those at $t=10^{5} \mathrm{yr}$. The main differences with the models at $t=10^{5} \mathrm{yr}$, however, are the water abundances of the models without X-rays. $\mathrm{H}_{2} \mathrm{O}$ is gradually destroyed in reactions with $\mathrm{HCO}^{+}$for temperatures $T \gtrsim 100 \mathrm{~K}$. In models without X-rays, $\mathrm{HCO}^{+}$is mainly formed by cosmic-ray induced reactions. At $t=10^{6} \mathrm{yr}$, water between $T \approx 100 \mathrm{~K}$ and $T \approx 200 \mathrm{~K}$ is destroyed down to abundances of a few $\times 10^{-7}$. The water abundances for $T<100 \mathrm{~K}$ on the other hand, are slightly higher compared to the models at $t=10^{5} \mathrm{yr}$. This is due to the relatively slow ion-molecule reactions that need time to build up water.

\subsection{Chemical reactions relevant for $\mathrm{H}_{2} \mathrm{O}$}

Water is assumed to be initially frozen out on the dust grains for temperatures $T<100 \mathrm{~K}$ and injected into the gas at $T=100 \mathrm{~K}$ with a fractional abundance $x\left(\mathrm{H}_{2} \mathrm{O}\right)=1.5 \times 10^{-4}$. In the presence of X-rays, water is then mainly destroyed in reactions with $\mathrm{H}_{3}^{+}$and $\mathrm{HCO}^{+}$. Another destruction route is the dissociation of water due to X-ray induced FUV photons. To estimate the importance of this reaction on the $\mathrm{H}_{2} \mathrm{O}$ abundance, models have been run for different $\mathrm{X}$-ray luminosities ignoring this reaction. The resulting $\mathrm{H}_{2} \mathrm{O}$ abundances vary by less than $15 \%$ in comparison to models where this reaction is included. It emphasizes that the main destruction reactions of $\mathrm{H}_{2} \mathrm{O}$ are the ion-molecule reactions with $\mathrm{H}_{3}^{+}$and $\mathrm{HCO}^{+}$, respectively. The bulk of the $\mathrm{H}_{2} \mathrm{O}$ not evaporated from the grains stems from the reaction of $\mathrm{OH}$ with $\mathrm{H}_{2}$, which becomes dominant for temperatures $T \gtrsim 250 \mathrm{~K}$, and from electron recombination reactions of $\mathrm{H}_{3} \mathrm{O}^{+}$. The adopted branching ratios for the dissociative recombination of $\mathrm{H}_{3} \mathrm{O}^{+}$to $\mathrm{H}_{2} \mathrm{O}$ and $\mathrm{OH}$ are 0.3 and 0.7 , respectively (e.g., Jensen et al. 2000). $\mathrm{H}_{3} \mathrm{O}^{+}$is abundantly produced in the reactions of water with $\mathrm{H}_{3}^{+}$and $\mathrm{HCO}^{+}$in the inner region of the envelope. Further away from the source, $\mathrm{H}_{3} \mathrm{O}^{+}$is most efficiently formed in the following way: $\mathrm{H}_{3}^{+}$reacts with atomic oxygen to produce $\mathrm{OH}^{+}$. This is followed by a series of hydrogen abstract reactions with $\mathrm{H}_{2}$ leading to $\mathrm{H}_{3} \mathrm{O}^{+}$. The main source for $\mathrm{H}_{3}^{+}$is the reaction of molecular hydrogen with $\mathrm{H}_{2}^{+}$, which is in predominantly produced by electron impact ionization of $\mathrm{H}_{2} . \mathrm{HCO}^{+}$is produced via the reaction of $\mathrm{H}_{3}^{+}$with $\mathrm{CO}$. $\mathrm{OH}$ is largely produced in recombination reactions of $\mathrm{H}_{3} \mathrm{O}^{+}$and destroyed in reactions with atomic oxygen or molecular hydrogen. The chemical network with reactions and species relevant for $\mathrm{H}_{2} \mathrm{O}$ in the X-ray models is presented in Fig. 5. Photoionizations and dissociations due to inner or outer FUV fields are not shown. 


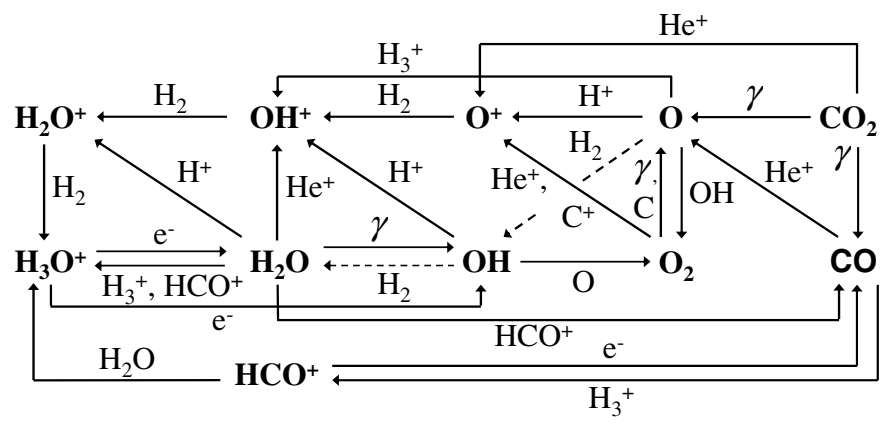

Fig. 5. Most important reactions involved in the X-ray chemistry of $\mathrm{H}_{2} \mathrm{O}$. Dashed lines indicate that the reaction is only important for temperatures $T \gtrsim 250 \mathrm{~K}$. $\gamma$ denotes the X-ray induced FUV photons. The reactions induced by central or interstellar FUV fields are not included.

\section{Applications to protostellar envelopes}

In order to study the influence of X-rays on the gas-phase water abundance in more realistic environments, the models are applied to envelopes of Class $\mathrm{I}\left(t \approx 10^{5}-10^{6} \mathrm{yr}\right)$ and Class 0 $\left(t \approx \mathrm{a}\right.$ few $\left.\times 10^{3}-10^{4} \mathrm{yr}\right)$ low-mass YSOs. These regions may be characterized by a power-law density distribution and an increasing gas temperature towards the central protostar. Class 0 and I envelopes mainly differ in their total mass. Typical envelope masses of Class 0 objects are $M_{\text {env }} \gtrsim 0.5 M_{\odot}$ whereas Class I sources have less massive envelopes with $M_{\text {env }} \lesssim 0.5 M_{\odot}$ (e.g., Jørgensen et al. 2002, 2005a). Although spherical 1D models are an approximation to the real geometry of protostellar cores, they successfully explain many of the observed features of lowmass protostars from few hundred AU out to $10^{4} \mathrm{AU}$ scales (e.g., Schöier et al. 2004; Jørgensen et al. 2002, 2005c) and provide a framework for discussing their overall chemical structure (e.g., Doty et al. 2004; Maret et al. 2004; Jørgensen et al. 2004). Inside a few hundred AU, however, the geometry is likely to be much more complex with cavities and a flattened protostellar disk in addition to the spherical inner envelope. Since no realistic physical models based on observations on those scales are yet available, we adopt the spherical models extrapolated to small radii as a starting point. Possible effects due to cavities and non-spherical symmetry are discussed throughout this section and in Sect. 4.

In the following models, the $\mathrm{H}_{2} \mathrm{O}$ chemistry is assumed to be irradiated by X-rays and FUV photons from the central source. The FUV models have been described in detail by Stäuber et al. (2004).

\subsection{Class I envelope model}

The density and temperature profiles adopted for a prototypical Class I source are presented in Fig. 6 and Table 2, based on the observations by Jørgensen et al. (2002). In that analysis, it was assumed that the gas temperature equals the dust temperature. X-rays and FUV fields, however, are able to increase the gas temperature due to photoelectric heating. The gas temperature has therefore been explicitly calculated for the different X-ray and FUV fluxes. The X-ray luminosity is varied between $L_{\mathrm{X}}=10^{26}-10^{32} \mathrm{erg} \mathrm{s}^{-1}$ and the FUV field from the central source $\left(G_{0, \text { in }}\right)$ at the inner radius from $10^{5}$ to $10^{8}$ with respect to the average ISRF. The heating rate due to X-rays is taken from Maloney et al. (1996), and for the FUV heating we follow Bakes $\&$ Tielens (1994). The heating rates are then compared to the cooling rate due to gas-dust collisions provided by Hollenbach \& McKee (1989). At lower densities $\left(n_{\mathrm{H}} \approx 10^{5} \mathrm{~cm}^{-3}\right)$ line cooling

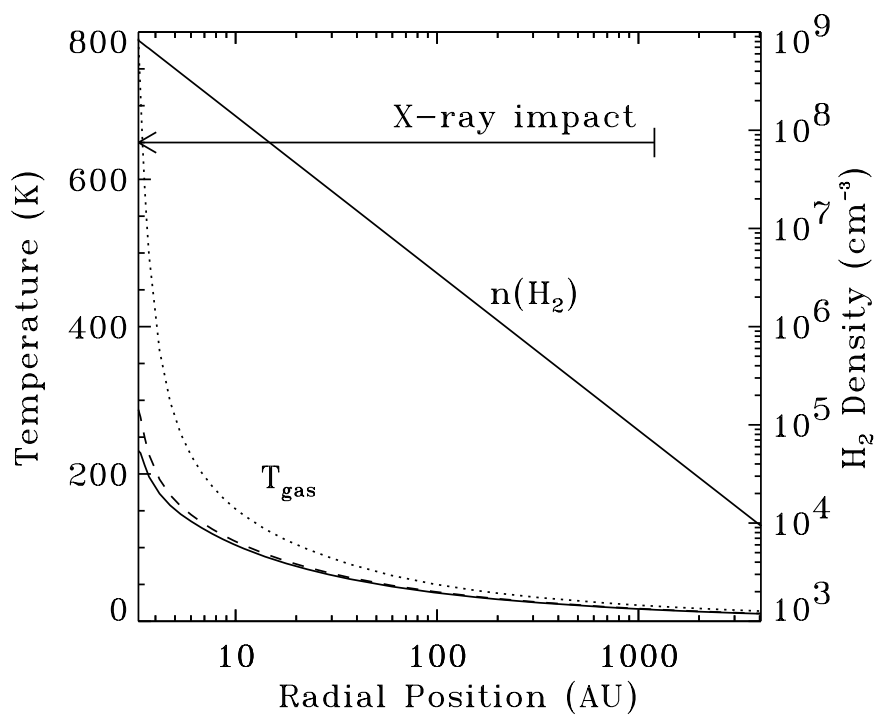

Fig. 6. Density and thermal structure for a prototypical Class I object adopted from the model results of Jørgensen et al. (2002) (solid lines). The dashed and dotted lines refer to the gas temperature for the models including an additional inner source of X-ray luminosity $\left(L_{\mathrm{X}}=\right.$ $10^{31} \mathrm{erg} \mathrm{s}^{-1}$ and $L_{\mathrm{X}}=10^{32} \mathrm{erg} \mathrm{s}^{-1}$, respectively). The arrow indicates the distance out to which $\mathrm{X}$-rays dominate the $\mathrm{H}_{2}$ ionization rate over cosmic-rays $\left(\zeta_{\mathrm{cr}}=0.8 \times 10^{-17} \mathrm{~s}^{-1}\right)$ for $L_{\mathrm{X}}=10^{30} \mathrm{erg} \mathrm{s}^{-1}$.

Table 2. Model parameters for a prototypical Class I object.

\begin{tabular}{ll}
\hline \hline$L_{\text {bol }}$ & $0.66 L_{\odot}$ \\
Envelope parameters: & \\
\hline Inner radius $r_{\text {in }}(T=250 \mathrm{~K})$ & $3.1 \mathrm{AU}$ \\
Outer radius $r_{\text {out }}(T=10 \mathrm{~K})$ & $4.3 \times 10^{3} \mathrm{AU}$ \\
Density at $1000 \mathrm{AU}, n\left(\mathrm{H}_{2}\right)$ & $8.8 \times 10^{4} \mathrm{~cm}^{-3}$ \\
Density power-law index, $p$ & 1.6 \\
Gas mass $M_{\text {env }}(T=10 \mathrm{~K})$ & $0.034 M_{\odot}$ \\
Column density $N_{\mathrm{H}_{2}}$ & $6.9 \times 10^{22} \mathrm{~cm}^{-2}$ \\
\hline
\end{tabular}

due to $\mathrm{C}^{+}, \mathrm{O}$ and $\mathrm{CO}$ also becomes important (see also Doty \& Neufeld 1997), but these effects are minor for our purpose since the densities in the regions of interest are always $n_{\mathrm{H}} \gtrsim 10^{6} \mathrm{~cm}^{-3}$.

It is found that only X-ray luminosities $L_{\mathrm{X}} \gtrsim 10^{31} \mathrm{erg} \mathrm{s}^{-1}$ lead to a noticeable increase in the gas temperature $\left(T_{\text {gas }}-T_{\text {dust }} \gtrsim\right.$ $10 \mathrm{~K})$. The results for $L_{\mathrm{X}}=10^{31} \mathrm{erg} \mathrm{s}^{-1}$ and $L_{\mathrm{X}}=10^{32} \mathrm{erg} \mathrm{s}^{-1}$ are plotted in Fig. 6 . The temperature of the gas is only affected in the inner $\approx 0.1 \mathrm{AU}$ by the central FUV field (not shown in Fig. 6). High FUV fields $\left(G_{0, \text { in }} \gtrsim 10^{7}\right)$ are needed though to increase the gas temperature significantly above the dust temperature.

The effects of the outer FUV field can be neglected. The optical depth in the region of interest $(T \gtrsim 100 \mathrm{~K})$ is $A_{\mathrm{V}} \approx 18$ and all FUV photons should be absorbed by the outer envelope for typical values of the ISRF. The outer FUV field is taken to be $G_{0, \text { out }}=1$ according to the average ISRF.

The adopted value of the cosmic-ray ionization rate $\zeta_{\mathrm{cr}}=$ $0.8 \times 10^{-17} \mathrm{~s}^{-1}$ is based on the results of Stäuber et al. (2005) who showed that low- $J$ lines of $\mathrm{HCO}^{+}$tend to trace the cosmicray ionization rate, whereas high- $J$ lines are more sensitive to the ionizing inner X-ray flux. To constrain $\zeta_{\text {cr }}$, the rate is varied in our models in order to fit the $\mathrm{HCO}^{+} 1-0$ observations toward the low-mass star-forming region Taurus (TMC 1) of Hogerheijde et al. (1997). It is found that $\zeta_{\text {cr }}=0.8 \times 10^{-17} \mathrm{~s}^{-1}$ fits the observations best. This value is at the lower end of the rates found 


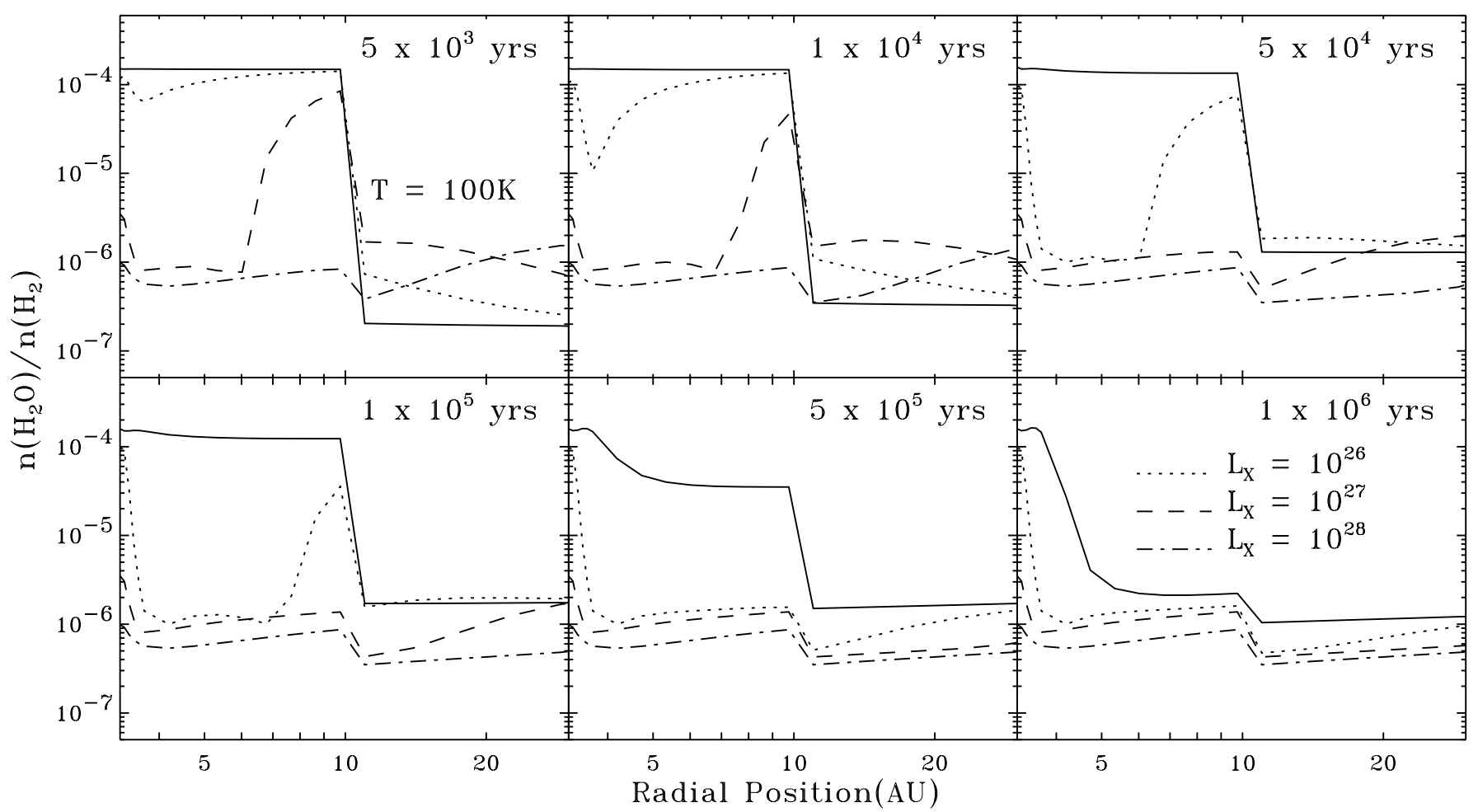

Fig. 7. Depth dependent temporal evolution of $\mathrm{H}_{2} \mathrm{O}$ for different $\mathrm{X}$-ray luminosities. The solid line corresponds to the model without X-rays. X-ray luminosities are given in $\operatorname{erg~s}^{-1}$. The $T=100 \mathrm{~K}$ mark is at $\approx 10 \mathrm{AU}$.

by van der Tak \& van Dishoeck (2000) toward high-mass starforming regions $\left(\zeta_{\mathrm{cr}}=0.61-5.6 \times 10^{-17} \mathrm{~s}^{-1}\right)$.

\subsection{Results for the Class I model}

\subsubsection{X-ray models}

The depth dependent water profiles for $t=5 \times 10^{3} \mathrm{yr}$ to $t=10^{6} \mathrm{yr}$ and for X-ray luminosities $L_{\mathrm{X}}=10^{26}-10^{28} \mathrm{erg} \mathrm{s}^{-1}$ are presented in Fig. 7. The inner FUV field is neglected in these models. It can be seen that an X-ray luminosity of $L_{\mathrm{X}} \gtrsim 10^{28} \mathrm{erg} \mathrm{s}^{-1} \mathrm{de}-$ stroys the gas-phase $\mathrm{H}_{2} \mathrm{O}$ within less than $5000 \mathrm{yr}$ from initial $x\left(\mathrm{H}_{2} \mathrm{O}\right) \approx 10^{-4}$ down to $x\left(\mathrm{H}_{2} \mathrm{O}\right) \approx 10^{-6}$ with respect to molecular hydrogen. For an X-ray luminosity of $L_{\mathrm{X}} \approx 10^{27} \mathrm{erg} \mathrm{s}^{-1}$ the timescale to destroy water is $t \gtrsim 5 \times 10^{4} \mathrm{yr}$. In models with $L_{\mathrm{X}} \lesssim$ $10^{26} \mathrm{erg} \mathrm{s}^{-1}$, water has still high abundances $\left(x\left(\mathrm{H}_{2} \mathrm{O}\right) \approx 10^{-4}\right)$ in the innermost part of the envelope. We therefore conclude that $\mathrm{X}$-ray luminosities $L_{\mathrm{X}} \gtrsim 10^{27} \mathrm{erg} \mathrm{s}^{-1}$ destroy all gas-phase water down to fractional abundances of $x\left(\mathrm{H}_{2} \mathrm{O}\right) \approx 10^{-6}$ on timescales $t \approx 5 \times 10^{4} \mathrm{yr}$ and $L_{\mathrm{X}} \gtrsim 10^{28} \mathrm{erg} \mathrm{s}^{-1}$ on a timescale of a few $\times 10^{3} \mathrm{yr}$.

The water profile for $t=10^{5} \mathrm{yr}$ and higher X-ray luminosities is shown in Fig. 8. The fractional abundances increase from $x\left(\mathrm{H}_{2} \mathrm{O}\right) \approx 10^{-6}$ to $x\left(\mathrm{H}_{2} \mathrm{O}\right) \approx 10^{-5}$ for increasing $\mathrm{X}$-ray luminosities between $L_{\mathrm{X}}=10^{29}-10^{31} \mathrm{erg} \mathrm{s}^{-1}$, and $x\left(\mathrm{H}_{2} \mathrm{O}\right)$ reaches even $\gtrsim 10^{-4}$ in the innermost part of the envelope for $L_{X}=$ $10^{32} \mathrm{erg} \mathrm{s}^{-1}$. This effect can be explained by the fast electron recombination reaction of $\mathrm{H}_{3} \mathrm{O}^{+}$that forms $\mathrm{H}_{2} \mathrm{O}$ and by the increasing gas temperature with increasing $\mathrm{X}$-ray flux: the reaction of $\mathrm{OH}$ with $\mathrm{H}_{2}$, that drives oxygen into $\mathrm{H}_{2} \mathrm{O}$, becomes faster than the destruction of water by $\mathrm{X}$-rays at higher temperatures, hence more water is produced for higher $\mathrm{X}$-ray fluxes. In the region $T<100 \mathrm{~K}, x\left(\mathrm{H}_{2} \mathrm{O}\right)$ decreases with increasing X-ray luminosity for $t=10^{5} \mathrm{yr}$, until $L_{\mathrm{X}} \approx 10^{29} \mathrm{erg} \mathrm{s}^{-1}$. Higher X-ray

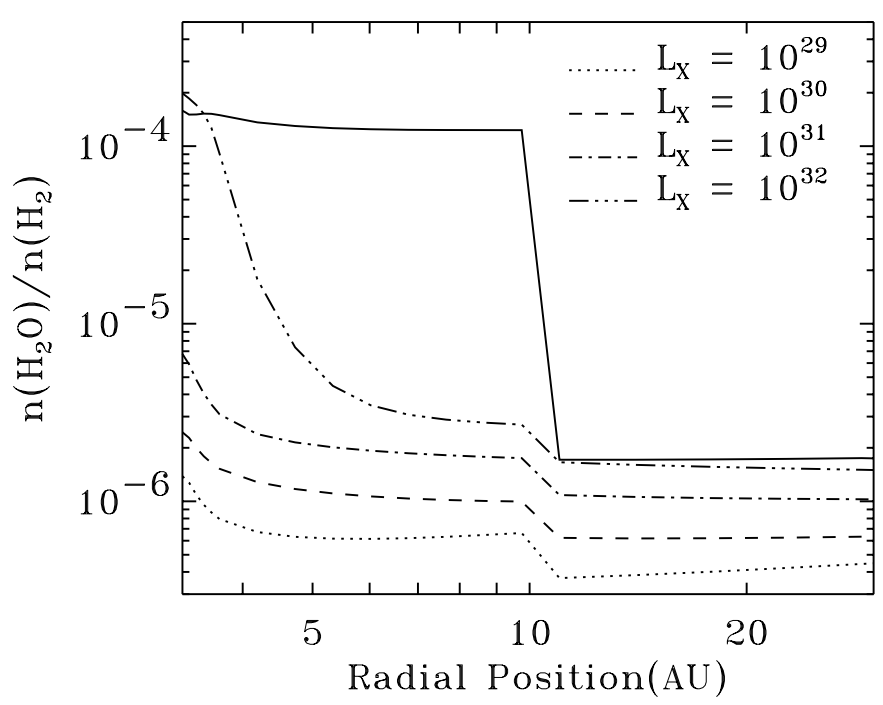

Fig. 8. Depth dependent fractional abundances of $\mathrm{H}_{2} \mathrm{O}$ for high X-ray luminosities and $t=10^{5} \mathrm{yr}$. The solid line corresponds to the model without X-rays. X-ray luminosities are given in $\operatorname{erg~s}^{-1}$.

luminosities lead to higher water abundances. This is again mainly due to the recombination of $\mathrm{H}_{3} \mathrm{O}^{+}$, which is more abundant in models with high $\mathrm{X}$-ray fluxes (see also Sect. 2). Figure 8 shows that $x\left(\mathrm{H}_{2} \mathrm{O}\right)$ increases from $\approx 4-5 \times 10^{-7}$ to $\approx 2 \times 10^{-6}$ with increasing $\mathrm{X}$-ray luminosity $\left(L_{\mathrm{X}} \gtrsim 10^{29} \mathrm{erg} \mathrm{s}^{-1}\right)$. At early times, the recombination of $\mathrm{H}_{3} \mathrm{O}^{+}$in the models with $\mathrm{X}$-rays leads to even higher $\mathrm{H}_{2} \mathrm{O}$ abundances in the region $T<100 \mathrm{~K}$ compared with the model without X-rays (Fig. 7).

The $\mathrm{OH}$ abundance profiles for different $\mathrm{X}$-ray luminosities at $t=10^{5} \mathrm{yr}$ are given in Fig. 9. The $\mathrm{OH}$ densities are normalized by $n\left(\mathrm{H}_{2}\right)$ (left) and by $n\left(\mathrm{H}_{2} \mathrm{O}\right)$ (right), respectively. In 


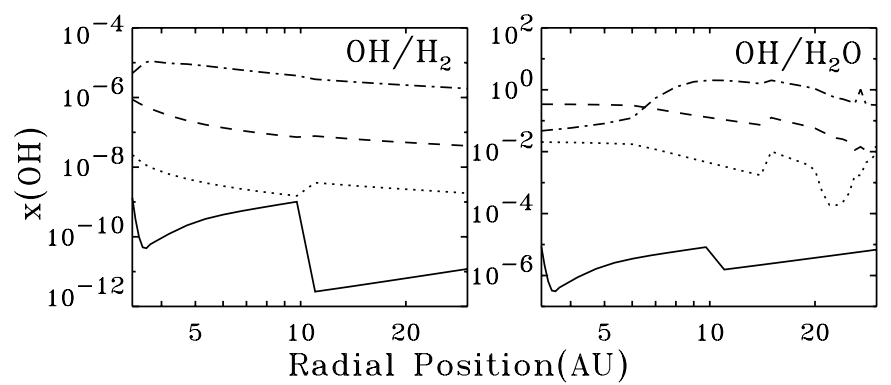

Fig. 9. Fractional abundances of $\mathrm{OH}\left(\right.$ left: $x(\mathrm{OH})=n(\mathrm{OH}) / n\left(\mathrm{H}_{2}\right)$, right: $x(\mathrm{OH})=n(\mathrm{OH}) / n\left(\mathrm{H}_{2} \mathrm{O}\right)$ ) for the model without X-rays (solid line), for $L_{\mathrm{X}}=10^{28} \mathrm{erg} \mathrm{s}^{-1}$ (dotted line), for $L_{\mathrm{X}}=10^{30} \mathrm{erg} \mathrm{s}^{-1}$ (dashed line) and for $L_{\mathrm{X}}=10^{32} \mathrm{erg} \mathrm{s}^{-1}$ (dashed-dotted line).

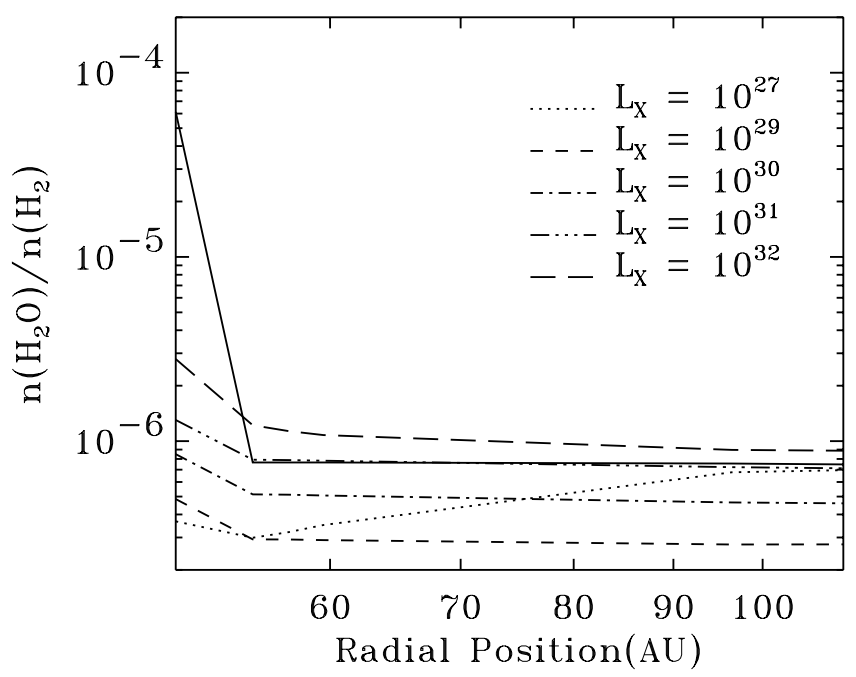

Fig. 10. Model with $r_{\text {in }}=50 \mathrm{AU}$ and $T\left(r_{\text {in }}\right)=100 \mathrm{~K}$ : depth dependent fractional abundances of $\mathrm{H}_{2} \mathrm{O}$ for different X-ray luminosities and $t=$ $10^{5} \mathrm{yr}$. The solid line corresponds to the model without X-rays. X-ray luminosities are given in $\operatorname{erg~s}^{-1}$.

the right part of Fig. 9 it can be seen that an X-ray luminosity $L_{\mathrm{X}}=10^{28} \mathrm{erg} \mathrm{s}^{-1}$ leads to $n(\mathrm{OH}) / n\left(\mathrm{H}_{2} \mathrm{O}\right) \approx 10^{-2}$, whereas $\mathrm{X}$-ray luminosities $L_{\mathrm{X}} \gtrsim 10^{30} \mathrm{erg} \mathrm{s}^{-1}$ can produce ratios of $n(\mathrm{OH}) / n\left(\mathrm{H}_{2} \mathrm{O}\right) \approx 0.1-1$ in the innermost part of the envelope. The $n(\mathrm{OH}) / n\left(\mathrm{H}_{2} \mathrm{O}\right)$ ratio in the hot $(T \gtrsim 100 \mathrm{~K})$ region of the envelope in the models without X-rays is only of the order of $\approx 10^{-4}$. X-rays therefore enhance the $\mathrm{OH}$ abundances as well as the $\mathrm{OH}$ to $\mathrm{H}_{2} \mathrm{O}$ abundance ratio.

The high densities $\left(n_{\mathrm{H}}>10^{8} \mathrm{~cm}^{-3}\right)$ and temperatures $(T>$ $200 \mathrm{~K})$ in the model at $r<10 \mathrm{AU}$ may be appropriate either to an inner envelope or to the inner regions of a circumstellar disk. However, there is also evidence for cavities in the inner few hundred AU (e.g., Jørgensen et al. 2005b). We have therefore also modeled an envelope where $r_{\text {in }}$ is assumed to be at $50 \mathrm{AU}$ with a temperature $T=100 \mathrm{~K}$. This corresponds to a source with $L_{\mathrm{bol}}=2 L_{\odot}$. The results for $t=10^{5} \mathrm{yr}$ are presented in Fig. 10. $\mathrm{H}_{2} \mathrm{O}$ is destroyed by the $\mathrm{X}$-rays and has abundances $x\left(\mathrm{H}_{2} \mathrm{O}\right)<10^{-5}$. At larger radii $\left(r_{\text {in }}>50 \mathrm{AU}\right)$ the dust will not be heated above $T>100 \mathrm{~K}$, i.e., the temperature where water evaporates into the gas phase, and the water abundances will stay below $x\left(\mathrm{H}_{2} \mathrm{O}\right)<10^{-5}$.

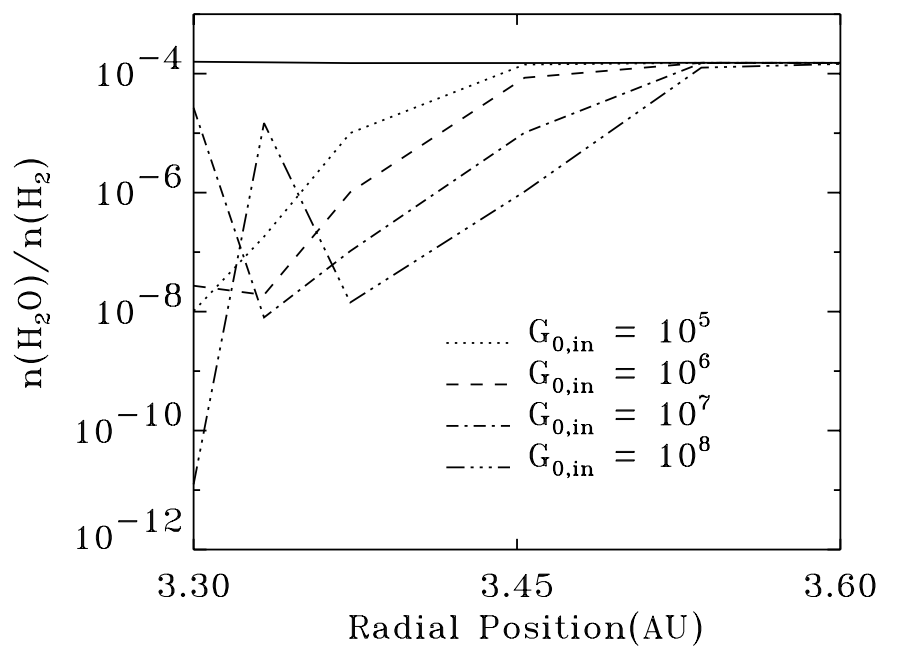

Fig. 11. Depth dependent fractional abundances of $\mathrm{H}_{2} \mathrm{O}$ for different inner FUV fields and $t=10^{5} \mathrm{yr}$. The solid line corresponds to the model with $G_{0, \text { in }}=0$. The X-ray emission is neglected.

\subsubsection{FUV models}

The $\mathrm{H}_{2} \mathrm{O}$ profiles for different inner FUV fields $\left(G_{0 \text {,in }}=10^{5}-10^{8}\right)$ are presented in Fig. 11 . The X-ray emission has been neglected in these models. As can be seen, inner FUV fields affect the water abundances only in the inner 0.2-0.3 AU. This is due to the high hydrogen densities $\left(n_{\mathrm{H}} \approx 10^{9} \mathrm{~cm}^{-3}\right)$ in this region that absorb the FUV photons very quickly. Although the FUV photons from the central source can destroy $\mathrm{H}_{2} \mathrm{O}$ in the innermost region, even very high FUV fields $\left(G_{0, \text { in }}=10^{8}\right)$ cannot destroy $\mathrm{H}_{2} \mathrm{O}$ out to $r_{100 \mathrm{~K}}=10 \mathrm{AU}$.

The FUV photons may reach distances in the envelope further away from the source by traveling through the outflow cones until they are scattered back into the envelope by dust grains (Spaans et al. 1995; Jørgensen 2004). However, the influence of the scattered photons is restricted to a small region around the outflow walls, since the FUV photons are absorbed quickly at these high densities and therefore water will not be destroyed on large scales.

Another possibility for the inner FUV field to affect larger distances might be a somewhat clumpy envelope. Models of clumpy photodissociation regions have shown that the FUV photons can travel deeper into the molecular cloud (Meixner \& Tielens 1993). At large radii with lower densities, the FUV photons primarily heat the gas and not the dust. $\mathrm{H}_{2} \mathrm{O}$ desorption will therefore not be much affected - but $\mathrm{H}_{2} \mathrm{O}$ gas may be photodissociated. Spaans \& van Dishoeck (2001) have shown that an inhomogeneous density distribution in molecular clouds lowers the column density of $\mathrm{H}_{2} \mathrm{O}$ by more than an order of magnitude compared with the homogeneous cloud model. The advent of ALMA or space-based far-infrared interferometers might help to investigate the possibility of clumpy envelopes in more detail, since a spatial resolution of $\lesssim 1^{\prime \prime}$ is needed to resolve the region of interest.

Models where a central X-ray source and circumstellar FUV fields were combined showed similar results for the influence of the FUV photons. It is thus concluded that X-rays clearly dominate the chemistry of the envelope in comparison to FUV fields. 

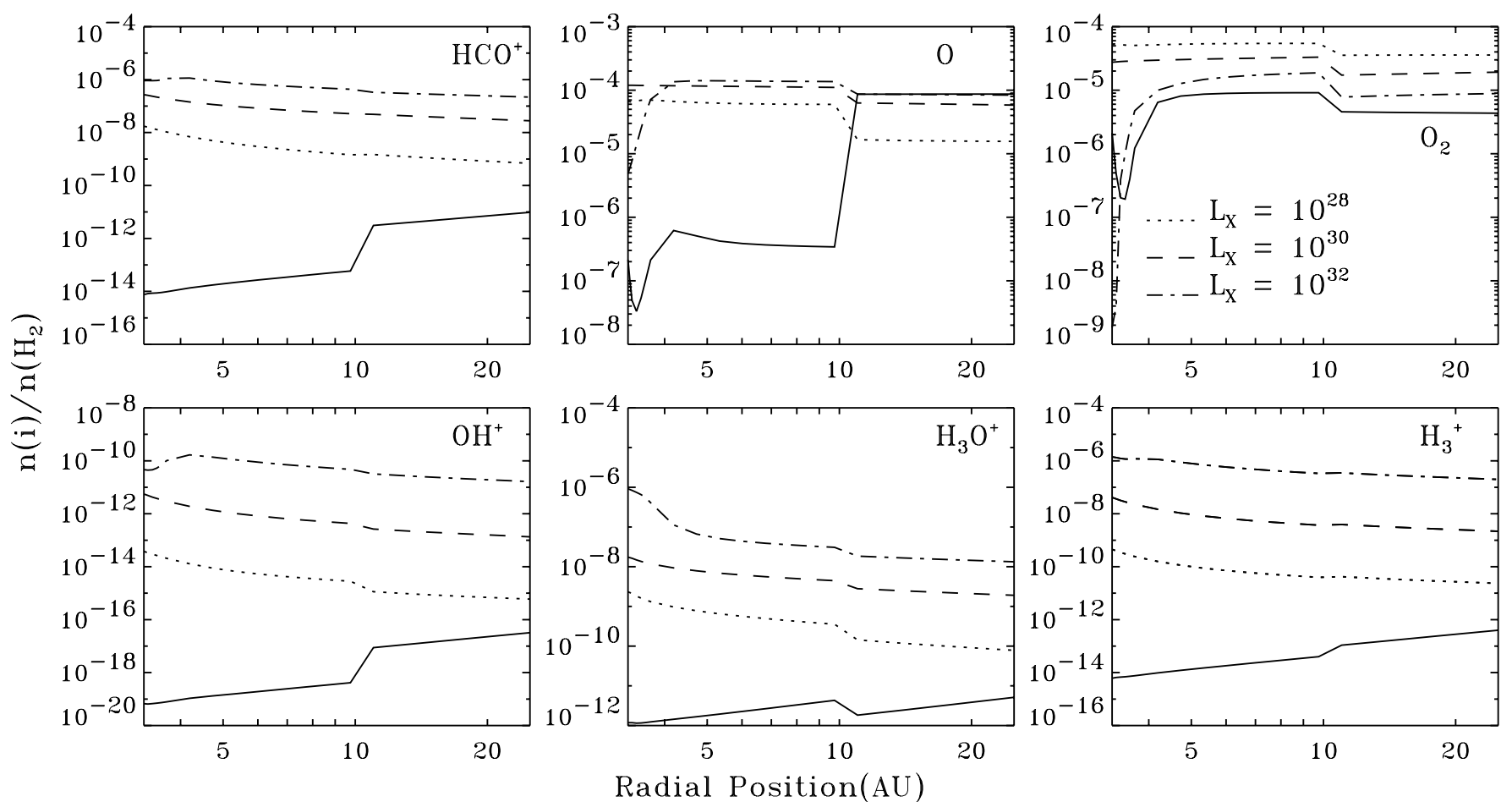

Fig. 12. Depth dependent fractional abundances of relevant species in the chemical $\mathrm{H}_{2} \mathrm{O}$ reaction network for different $\mathrm{X}$-ray luminosities and $t=10^{5} \mathrm{yr}$. The solid line corresponds to the model without X-rays or inner FUV fields. X-ray luminosities are given in erg s $\mathrm{s}^{-1}$.

\subsubsection{Other species}

Depth dependent fractional abundances of the main species involved in the water reaction network are presented in Fig. 12 for $t=10^{5} \mathrm{yr}$. Atomic oxygen is mainly produced by the X-ray induced FUV dissociation of $\mathrm{O}_{2}$ and the dissociative ionization of $\mathrm{CO}$ by $\mathrm{He}^{+}$. Atomic oxygen reaches fractional abundances of $x(\mathrm{O}) \approx 10^{-4}$ in the inner warm $(T>100 \mathrm{~K})$ region. The $\mathrm{OH}^{+}$abundance is dramatically increased by X-rays (see also Stäuber et al. 2005). The fractional abundance of $\mathrm{OH}^{+}$is $\approx 10^{-14}$ for $L_{\mathrm{X}}=10^{28} \mathrm{erg} \mathrm{s}^{-1}, x\left(\mathrm{OH}^{+}\right) \approx 10^{-12}$ for $L_{\mathrm{X}}=10^{30} \mathrm{erg} \mathrm{s}^{-1}$ and $x\left(\mathrm{OH}^{+}\right) \approx 10^{-10}$ for $L_{\mathrm{X}}=10^{32} \mathrm{erg} \mathrm{s}^{-1}$ in the inner region of the envelope. It is mainly produced in the ion-molecule reactions of $\mathrm{O}$ with $\mathrm{H}_{3}^{+}, \mathrm{O}^{+}$with $\mathrm{H}_{2}$ and $\mathrm{OH}$ with $\mathrm{H}^{+}$.

$\mathrm{O}_{2}$ has fractional abundances $x\left(\mathrm{O}_{2}\right) \approx 10^{-5}-10^{-4}$ in the models, much higher than observed. Molecular oxygen is efficiently produced in the reaction of atomic oxygen with $\mathrm{OH}$. There is an uncertainty in the rate of this reaction for $\mathrm{O}$ in the ground fine-structure level $\left({ }^{3} \mathrm{P}_{2}\right)$, however, and the rate coefficient may be lower than that cited by UMIST (Sims et al. 2006). $\mathrm{O}_{2}$ is mainly destroyed in the reaction with $\mathrm{H}_{3}^{+}$and by X-ray induced FUV photons. $\mathrm{O}_{2}$ has been searched with SWAS (e.g., Melnick 2004) and ODIN (e.g., Pagani et al. 2003) but has not been reported in the literature to date. Derived upper limits on $\mathrm{O}_{2}$ column densities are usually of the order of $\approx$ a few $\times 10^{15} \mathrm{~cm}^{-2}$ or $x\left(\mathrm{O}_{2}\right) \approx \mathrm{a}$ few $\times 10^{-7}$. Standard chemical models normally overestimate this value by 2-3 orders of magnitude (see Melnick 2004; Pagani et al. 2003 for a more detailed discussion). Our models are no exception. However, the $30^{\prime \prime}$ averaged column density in the Class I models are $N_{\text {beam }}\left(\mathrm{O}_{2}\right) \lesssim 10^{15} \mathrm{~cm}^{-2}$, which is well within the upper limits derived by SWAS and ODIN. Oxygen could be frozen out on grains in some form at lower temperatures and therefore not seen with the big beams of ODIN $\left(9^{\prime}\right)$ and SWAS $\left(3.5^{\prime} \times 5.0^{\prime}\right)$. Herschel might shed some light on this
Table 3. Radial column densities of $\mathrm{H}_{3}^{+}$for the Class I source.

\begin{tabular}{cccc}
\hline \hline $\begin{array}{c}\log \left(L_{\mathrm{X}}\right) \\
\log \left(\mathrm{erg} \mathrm{s}^{-1}\right)\end{array}$ & $\begin{array}{c}N_{\text {rad }} \\
\mathrm{cm}^{-2}\end{array}$ & $\begin{array}{c}\log \left(L_{\mathrm{X}}\right) \\
\log \left(\mathrm{erg} \mathrm{s}^{-1}\right)\end{array}$ & $\begin{array}{c}N_{\text {rad }} \\
\mathrm{cm}^{-2}\end{array}$ \\
\hline 0 & $8.0 \mathrm{E} 11(2.0 \mathrm{E} 09)$ & 30 & $4.8 \mathrm{E} 14(4.2 \mathrm{E} 11)$ \\
27 & $1.3 \mathrm{E} 12(2.5 \mathrm{E} 09)$ & 31 & $4.5 \mathrm{E} 15(3.9 \mathrm{E} 12)$ \\
28 & $5.9 \mathrm{E} 12(6.5 \mathrm{E} 09)$ & 32 & $3.6 \mathrm{E} 16(2.9 \mathrm{E} 13)$ \\
29 & $5.0 \mathrm{E} 13(4.5 \mathrm{E} 10)$ & & \\
\hline
\end{tabular}

matter with its relatively small beam widths (8-35", depending on frequency).

$\mathrm{H}_{3}^{+}$is a key molecule in the chemical reaction network of water and its abundance is greatly enhanced in the models including $\mathrm{X}$-rays. Thus, observations of $\mathrm{H}_{3}^{+}$in absorption at $\approx 3.7 \mu \mathrm{m}$ (e.g., McCall et al. 1999) would allow a direct test of the models. The integrated radial column densities $N_{\text {rad }}$ of $\mathrm{H}_{3}^{+}$are therefore presented for the Class I X-ray models in Table 3 . The values given in parenthesis are $\mathrm{H}_{3}^{+}$column densities calculated for the model with $r_{\text {in }}=50 \mathrm{AU}$, i.e. the model with a protostellar hole.

\subsection{Class 0 envelope model}

Table 4 lists the main parameters for a prototypical Class 0 envelope model (Schöier et al. 2002). The crucial differences from the modeling point of view between the Class 0 and I envelope are the much higher bolometric luminosity, envelope mass and column density of the younger object, that lead to a different density and temperature distribution. The initial chemical abundances are again taken as given in Table 1. Freeze-out of $\mathrm{CO}$ is taken into account for temperatures $T<20 \mathrm{~K}$. The increased gas temperature due to X-rays has been calculated as described in Sect. 3.1. The X-ray luminosity is varied between $L_{\mathrm{X}}=10^{28}-10^{32} \mathrm{erg} \mathrm{s}^{-1}$. 
Table 4. Model parameters for a prototypical Class 0 source.

\begin{tabular}{ll}
\hline \hline$L_{\text {bol }}$ & $27 L_{\odot}$ \\
Envelope parameters: & \\
\hline Inner radius $r_{\text {in }}$ & $32 \mathrm{AU}$ \\
Outer radius $r_{\text {out }}$ & $8 \times 10^{3} \mathrm{AU}$ \\
Density at $1000 \mathrm{AU}, n\left(\mathrm{H}_{2}\right)$ & $6.7 \times 10^{6} \mathrm{~cm}^{-3}$ \\
Density power-law index, $p$ & 1.7 \\
Mass $M_{\text {env }}$ & $5.4 M_{\odot}$ \\
Column density $N_{\mathrm{H}_{2}}$ & $1.6 \times 10^{24} \mathrm{~cm}^{-2}$ \\
\hline
\end{tabular}

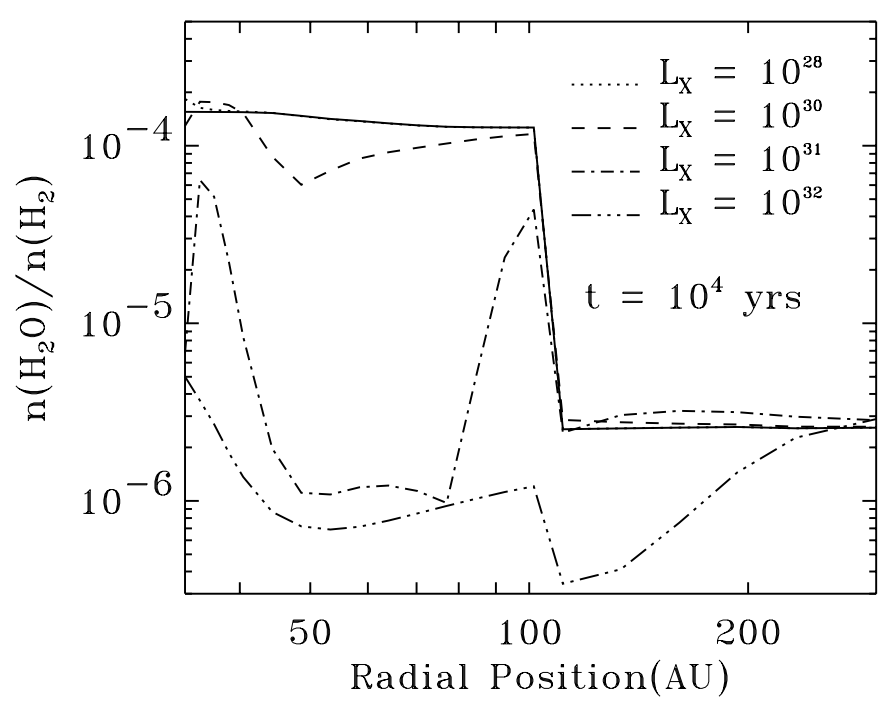

Fig. 13. Depth dependent fractional abundances of $\mathrm{H}_{2} \mathrm{O}$ for a Class 0 object for different X-ray luminosities and $t=10^{4} \mathrm{yr}$. The solid line corresponds to the model without X-rays. X-ray luminosities are given in $\operatorname{erg~s}^{-1}$.

The results for $\mathrm{H}_{2} \mathrm{O}$ are shown in Fig. 13 for $t=10^{4} \mathrm{yr}$ and in Fig. 14 for $t=10^{5} \mathrm{yr}$. At $t=10^{4} \mathrm{yr}$, only high X-ray luminosities $\left(L_{\mathrm{X}} \gtrsim 10^{32} \mathrm{erg} \mathrm{s}^{-1}\right)$ lead to water abundances $x\left(\mathrm{H}_{2} \mathrm{O}\right) \lesssim 10^{-5}$. An $\mathrm{X}$-ray luminosity of $L_{\mathrm{X}} \gtrsim 10^{31} \mathrm{erg} \mathrm{s}^{-1}$ destroys water between $\approx 40-80$ AU with higher abundances $\left(x\left(\mathrm{H}_{2} \mathrm{O}\right) \approx \mathrm{a}\right.$ few $\left.\times 10^{-5}\right)$ at $\approx 35 \mathrm{AU}$ and $\approx 100 \mathrm{AU}$. Water is not significantly destroyed for X-ray luminosities $L_{\mathrm{X}} \lesssim 10^{30} \mathrm{erg} \mathrm{s}^{-1}$. Similar conclusions hold for $t=10^{5} \mathrm{yr}$ although the region where $x\left(\mathrm{H}_{2} \mathrm{O}\right) \gtrsim 10^{-5}$ is less than $50 \mathrm{AU}(T \gtrsim 250 \mathrm{~K})$. Compared to the Class I models (after appropriate scaling of the X-ray flux $\propto L_{X} r^{-2}$ ), water is less destroyed by $\mathrm{X}$-rays in the Class 0 envelope. The reason for this are the high densities $\left(n_{\mathrm{H}} \gtrsim 10^{9} \mathrm{~cm}^{-3}\right)$ in the innermost region of the Class 0 source that absorb the $\mathrm{X}$-ray flux.

\section{Discussion}

As discussed in Sect. 2, X-rays reduce the water abundance preferably in regions where the gas temperature is $T \lesssim 250-300 \mathrm{~K}\left(x\left(\mathrm{H}_{2} \mathrm{O}\right) \approx 10^{-6}\right)$. At low densities $\left(n_{\mathrm{H}} \lesssim\right.$ $\left.10^{5} \mathrm{~cm}^{-3}\right)$, the fractional water abundance is less than $\approx 10^{-7}$ for X-ray fluxes $F_{\mathrm{X}} \gtrsim 10 \mathrm{erg} \mathrm{s}^{-1} \mathrm{~cm}^{-2}$ even for high gas temperatures (Fig. 4). For higher densities and temperatures $T \gtrsim 250$ $300 \mathrm{~K}$, water has fractional abundances $x\left(\mathrm{H}_{2} \mathrm{O}\right) \gtrsim 10^{-4}$ (nearly) independent of the X-ray flux. In the following, we discuss these findings in the context of protostellar envelopes and other models.

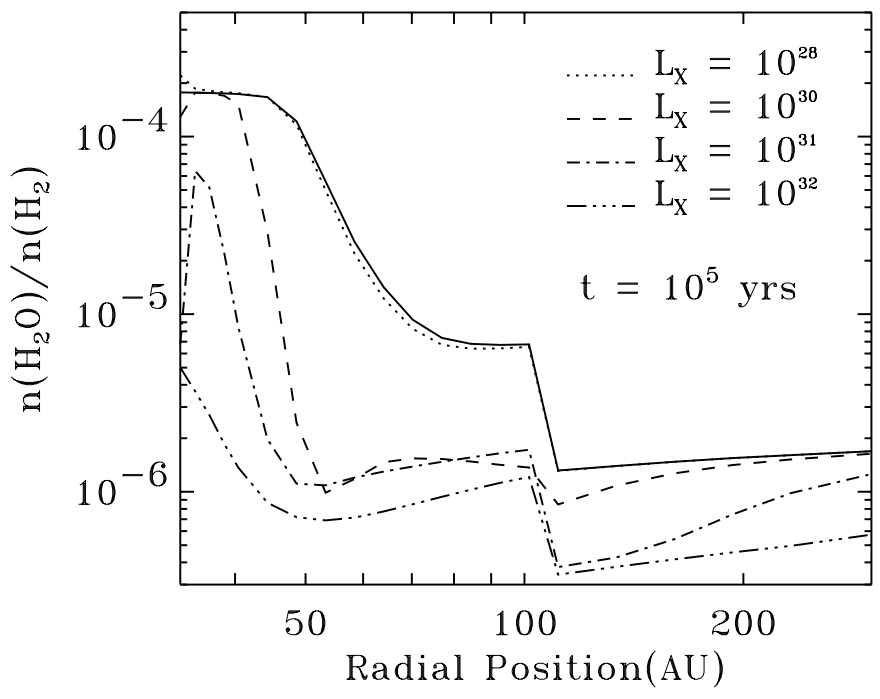

Fig. 14. Depth dependent fractional abundances of $\mathrm{H}_{2} \mathrm{O}$ for a Class 0 object for different X-ray luminosities and $t=10^{5} \mathrm{yr}$. The solid line corresponds to the model without $\mathrm{X}$-rays. $\mathrm{X}$-ray luminosities are given in $\mathrm{erg} \mathrm{s}^{-1}$.

\subsection{Envelope models}

For high-mass YSOs, the observed water abundance can be successfully explained within the hot-core scenario in which the high temperatures in the inner envelope drive most of the oxygen into water and in which most of the water is frozen out onto grains in the outer region (e.g., Doty et al. 2002; Boonman et al. 2003b). Recent interferometer studies of $\mathrm{H}_{2}^{18} \mathrm{O}$ by van der Tak et al. (2006) towards the high-mass young stellar object (YSO) AFGL 2591 confirm the existence of a hot $(T>100 \mathrm{~K})$ compact central region with high abundant water $\left(x\left(\mathrm{H}_{2} \mathrm{O}\right) \approx 10^{-4}\right)$ and a region with lower abundances in the colder outer part. Although X-ray models predict the destruction of water also in high-mass objects (Stäuber et al. 2005), these envelopes are likely to have regions with temperatures exceeding $T=250 \mathrm{~K}$ (van der Tak et al. 2000). In addition, the distance from the central source to the region in the envelope where the gas temperature is $T=100 \mathrm{~K}$, is much larger in high-mass sources compared to low-mass objects due to their high bolometric luminosities. The X-ray flux decreases rapidly with $r^{-2}$ and may already be too low to destroy $\mathrm{H}_{2} \mathrm{O}$. Water can therefore have fractional abundances of the order of $10^{-4}$ in envelopes around high-mass YSOs despite their possible X-ray emission.

Similar jumps in the water abundances are also observed toward low-mass Class 0 sources, although the derived abundances in the inner part are usually much lower compared to the massive objects. Ceccarelli et al. (2000) derived water abundances of $x_{\mathrm{in}}\left(\mathrm{H}_{2} \mathrm{O}\right)=3 \times 10^{-6}$ for the inner part of the envelope of the Class 0 source IRAS $16293-2422$ and $x_{\text {out }}\left(\mathrm{H}_{2} \mathrm{O}\right)=5 \times 10^{-7}$ for the outer part from detailed models of ISO observations. Maret et al. (2002) found $x_{\mathrm{in}}\left(\mathrm{H}_{2} \mathrm{O}\right)=5 \times 10^{-6}$ for the Class 0 object NGC1333-IRAS4 and $x_{\text {out }}\left(\mathrm{H}_{2} \mathrm{O}\right)=5 \times 10^{-7}$. Within the spherical envelope models in Sect. 3.3, such jumps with relatively low inner water abundances occur for high X-ray luminosities $L_{\mathrm{X}}=10^{31}-10^{32} \mathrm{erg} \mathrm{s}^{-1}$ and $t=10^{5} \mathrm{yr}$ (Fig. 14). X-rays are therefore a possible explanation for the low water abundances found in these objects.

Class I envelopes may have no or only a small region where the temperature exceeds $T \approx 250 \mathrm{~K}$. The inner part of the envelope may already have been dispersed by outflows or accreted 
onto the star/disk system. Water is therefore easily destroyed by X-rays. According to the models in Sect. 3.2.1, the average water abundance is predicted to be at most $x\left(\mathrm{H}_{2} \mathrm{O}\right) \approx 10^{-6}$ in Class I sources with X-ray luminosities $L_{\mathrm{X}} \gtrsim 10^{27} \mathrm{erg} \mathrm{s}^{-1}$. Note that the generic Class I model in Sect. 3.1 is considered as an extreme case where the gas temperature gets $T \approx 250 \mathrm{~K}$ close to the protostar. If the inner envelope has cavities of any kind, the gas temperature of the envelope will be lower at the inner edge of the envelope $\left(r_{\text {in }}\right)$, since this will be at a larger distance and since the dust temperature roughly falls with $r_{\text {in }}^{-0.4}$. Water will in that case be destroyed even more readily (Sect. 3.2.1, Fig. 10), unless there is some mechanism (e.g., photodesorption) that returns water ice to the gas phase.

\subsection{Disks and outflows}

As discussed in Sect. 3, the inner envelopes of Class 0 and I sources are likely to have a much more complex geometry than the spherical envelopes adopted here. If protostellar cavities or holes (Jørgensen et al. 2005b) are common, the emission from $\mathrm{H}_{2} \mathrm{O}$ and other typical "hot core" species may be dominated by the protostellar accretion disk or outflow hot spots rather than by the envelope. Disks are known to be present (e.g., Brown et al. 2000; Looney et al. 2000) and can dominate over the hot core in terms of column density (Jørgensen et al. 2005c). From the chemical point of view, the disk and hot core scenario are difficult to distinguish since both have high densities and temperatures in their inner region. According to the results in Sect. 2, the X-ray flux of a protostar with $L_{X}=10^{31} \mathrm{erg} \mathrm{s}^{-1}$ and an absorbing column density of $N_{\mathrm{H}}=10^{24} \mathrm{~cm}^{-2}$ at the outer part of a protostellar disk $(r \approx 400 \mathrm{AU})$ is $F_{\mathrm{X}} \approx 10^{-3} \mathrm{erg} \mathrm{s}^{-1} \mathrm{~cm}^{-2}$. Water at temperatures below $T \approx 250 \mathrm{~K}$ is therefore destroyed from initial $x\left(\mathrm{H}_{2} \mathrm{O}\right) \approx 10^{-4}$ down to $x\left(\mathrm{H}_{2} \mathrm{O}\right) \approx 10^{-7}-10^{-6}$. High column densities and/or temperatures are needed for the water to persist at $x\left(\mathrm{H}_{2} \mathrm{O}\right) \approx 10^{-4}$ in protostellar disks. For comparison, the chemistry disk models including X-rays by Aikawa \& Herbst (2001) have $\mathrm{H}_{2} \mathrm{O}$ abundances below $\approx 10^{-8}$ at $r=500 \mathrm{AU}$ from the central source. In the disk atmosphere models of Glassgold et al. (2004), water has fractional abundances $x\left(\mathrm{H}_{2} \mathrm{O}\right)<10^{-5}$ at $1 \mathrm{AU}$ from the source for vertical column densities of less than a few $\times 10^{23} \mathrm{~cm}^{-2}$.

Recent results presented by Chandler et al. (2005) for the inner $\approx 1^{\prime \prime}$ of IRAS $16293-2422$ suggest outflow shocks to be the origin for the hot core species. Giannini et al. (2001) and Nisini et al. (2002) pointed out the importance of outflows for the water abundances in Class 0 and I sources on larger scales. The results of Sect. 2 imply that the source of $\mathrm{H}_{2} \mathrm{O}$ emission with $x\left(\mathrm{H}_{2} \mathrm{O}\right) \approx 10^{-4}$ from outflow regions with gas temperatures $100 \mathrm{~K} \lesssim T \lesssim 250 \mathrm{~K}$ has to be located at a certain distance $d\left(L_{\mathrm{X}}\right)$ from the X-ray source in order to survive. Consider, for example, $t=10^{4} \mathrm{yr}$, typical outflow densities $\left(n_{\mathrm{H}}=10^{4}-10^{6} \mathrm{~cm}^{-3}\right)$ and an X-ray absorbing column density $N_{\mathrm{H}}=5 \times 10^{21} \mathrm{~cm}^{-2}$. It can then be concluded from Figs. 1-4 (Sect. 2), that the $\mathrm{H}_{2} \mathrm{O}$ emitting source would have to be at $d \gtrsim 170 \mathrm{AU}$ for $L_{\mathrm{X}}=10^{28} \mathrm{erg} \mathrm{s}^{-1}$, at $d \gtrsim 550 \mathrm{AU}$ for $L_{\mathrm{X}}=10^{29} \mathrm{erg} \mathrm{s}^{-1}$, at $d \gtrsim 1700 \mathrm{AU}$ for $L_{\mathrm{X}}=10^{30} \mathrm{erg} \mathrm{s}^{-1}$ and at $d \gtrsim 5500 \mathrm{AU}$ for $L_{\mathrm{X}}=10^{31} \mathrm{erg} \mathrm{s}^{-1}$. Larger hydrogen densities and X-ray absorbing column densities, however, decrease these distances. The physical quantities of the observed outflows by Benedettini et al. (2002) range between $300 \mathrm{~K} \lesssim T \lesssim 1400 \mathrm{~K}$ and $4 \times 10^{4} \mathrm{~cm}^{-3}$ $\lesssim n_{\mathrm{H}} \lesssim 2 \times 10^{6} \mathrm{~cm}^{-3}$. It is interesting to see, that the derived water abundances for $T>300 \mathrm{~K}$ by Benedettini et al. are a factor of $\approx 10$ higher than those at $T=300 \mathrm{~K}$. This is in good agreement with the results in Sect. 2 where the X-ray models show a jump at these densities but where the models without X-rays show no jump at $T=300 \mathrm{~K}$. X-rays are therefore also predicted to influence the water abundances in outflow hot spots. It should be noted that FUV fields from the central star may also influence the water abundances if the photons can impact the $\mathrm{H}_{2} \mathrm{O}$ emission region relatively unhindered, e.g. through outflow cones. High FUV fields tend to destroy water even at high temperatures though (Sect. 3.2.2; Stäuber et al. 2004).

\section{Conclusion}

The gas-phase water abundance is found to be critically dependent on time, gas temperature, hydrogen density and X-ray flux (Sect. 2). Three distinct regimes are identified: in the first regime $(T \lesssim 100 \mathrm{~K})$, the water abundance is $x\left(\mathrm{H}_{2} \mathrm{O}\right) \approx 10^{-7}-10^{-6}$ and can be somewhat enhanced or destroyed by X-rays in comparison to models without $\mathrm{X}$-rays. In the second regime $(250 \mathrm{~K}$ $\lesssim T \lesssim 100 \mathrm{~K})$, water is released from grains with $x\left(\mathrm{H}_{2} \mathrm{O}\right) \approx$ $10^{-4}$ but quickly reduced by X-rays to fractional abundances $x\left(\mathrm{H}_{2} \mathrm{O}\right) \approx 10^{-6}$. The third regime $(T \gtrsim 250 \mathrm{~K})$ is characterized by high water abundances $\left(x\left(\mathrm{H}_{2} \mathrm{O}\right) \gtrsim 10^{-4}\right)$ due to the efficient reaction of $\mathrm{OH}$ with molecular hydrogen. At low densities $\left(n_{\mathrm{H}} \lesssim 10^{5} \mathrm{~cm}^{-3}\right)$ water is destroyed even at high temperatures for X-ray fluxes $F_{\mathrm{X}} \gtrsim 10 \mathrm{erg} \mathrm{s}^{-1} \mathrm{~cm}^{-2}$. In general, higher gas temperatures and higher hydrogen densities allow higher $\mathrm{X}$-ray fluxes for $\mathrm{H}_{2} \mathrm{O}$ to survive. Water is mainly destroyed in reactions with the $\mathrm{X}$-ray enhanced species $\mathrm{HCO}^{+}$and $\mathrm{H}_{3}^{+}$. It is also destroyed by internally created FUV photons.

The envelopes of both Class I (Sect. 3.1) and Class 0 (Sect. 3.3) objects were modeled under the influence of central X-ray and FUV emission. The results for the Class I envelope show that an initial warm gas-phase water abundance of $x\left(\mathrm{H}_{2} \mathrm{O}\right) \approx 10^{-4}$ is reduced to $x\left(\mathrm{H}_{2} \mathrm{O}\right) \approx 10^{-6}$ within $\approx 5000 \mathrm{yr}$ for $\mathrm{X}$-ray luminosities $L_{\mathrm{X}} \gtrsim 10^{28} \mathrm{erg} \mathrm{s}^{-1}$ and within $\approx 5 \times 10^{4} \mathrm{yr}$ for X-ray luminosities $L_{\mathrm{X}} \gtrsim 10^{27} \mathrm{erg} \mathrm{s}^{-1}$, consistent with reported upper limits for $\mathrm{H}_{2} \mathrm{O}$ towards Class I sources. Water is also destroyed by $\mathrm{X}$-rays in the Class 0 object, but higher X-ray fluxes are needed due to the higher densities in the inner region. The influence of a central FUV field is negligible in our models, unless the FUV photons can escape to larger distances (Sect. 3.2.2).

The current protostellar models are limited to spherical symmetry, which is obviously an approximation to the true geometry of the inner few hundred AU where cavities and flattened disks may be present. Nevertheless, the high densities and temperatures in the current models are representative of such regions. In particular, X-rays are predicted to regulate the $\mathrm{H}_{2} \mathrm{O}$ abundances wherever the gas temperature is $T \lesssim 250-300 \mathrm{~K}$. This will not only be the case for $\mathrm{H}_{2} \mathrm{O}$ originating from envelopes or disks in high-mass star-forming regions, but also for $\mathrm{H}_{2} \mathrm{O}$ produced in outflow hot spots around low-mass objects. The presence of cavities or outflow cones will have the result that X-rays and/or FUV photons penetrate to larger radii in certain directions.

Future instruments such as Herschel Space Observatory will allow detailed studies of the water abundances in both high and low-mass YSOs. With its high resolution instrument HIFI (de Graauw \& Helmich 2001), it will be possible to distinguish shock heated $\mathrm{H}_{2} \mathrm{O}$ from $\mathrm{H}_{2} \mathrm{O}$ in hot cores or disks from their line profiles. Observations of the optically thin isotope $\mathrm{H}_{2}^{18} \mathrm{O}$ will help to sample the envelope, whereas optically thick lines may give information about outflow properties. PACS will delineate the water abundances on $9^{\prime \prime}$ scale including maps of the outflows. ALMA, on the other hand, will be able to resolve the innermost region $\left(<0.1^{\prime \prime}\right)$ of YSO envelopes and/or disks in order to study the chemistry and therefore its physical properties 
in molecules other than $\mathrm{H}_{2} \mathrm{O}$. Limited maps of $\mathrm{H}_{2}^{18} \mathrm{O}$ on arcsec scale may be possible though with ALMA under exceptional conditions in the $203 \mathrm{GHz}$ line. High- $J$ lines of $\mathrm{CO}$ will reveal the gas temperature in these regions whereas the observation of X-ray and FUV tracers (Stäuber et al. 2004, 2005) may help to clarify the high-energy properties of young stellar objects. Together, these new facilities and models will reveal much about the physical and chemical structure of the inner regions of young stellar objects, which are currently poorly understood.

Acknowledgements. This work was partially supported under grants from The Research Corporation (SDD). The research of JKJ was supported by NASA Origins Grant NAG5-13050. Astrochemistry in Leiden is supported by the Netherlands Research School for Astronomy (NOVA) and by a Spinoza grant from the Netherlands Organization for Scientific Research (NWO).

\section{References}

Aikawa, Y., \& Herbst, E. 2001, A\&A, 371, 1107

Bakes, E. L. O., \& Tielens, A. G. G. M. 1994, ApJ, 427, 822

Benedettini, M., Viti, S., Giannini, T., et al. 2002, A\&A, 395, 657

Boogert, A. C. A., Pontoppidan, K. M., Lahuis, F., et al. 2004, ApJS, 154, 359

Boonman, A. M. S., \& van Dishoeck, E. F. 2003, A\&A, 403, 1003

Boonman, A. M. S., van Dishoeck, E. F., Lahuis, F., \& Doty, S. D. 2003a, A\&A, 399,1063

Boonman, A. M. S., Doty, S. D., van Dishoeck, E. F., et al. 2003b, A\&A, 406, 937

Brown, D. W., Chandler, C. J., Carlstrom, J. E., et al. 2000, MNRAS, 319, 154

Casanova, S., Montmerle, T., Feigelson, E. D., \& André, P. 1995, ApJ, 439, 752

Ceccarelli, C., Hollenbach, D. J., \& Tielens, A. G. G. M. 1996, ApJ, 471, 400

Ceccarelli, C., Castets, A., Caux, E., et al. 2000, A\&A, 355, 1129

Chandler, C. J., Brogan, C. L., Shirley, Y. L., \& Loinard, L. 2005, ApJ, 632, 371

Charnley, S. B. 1997, ApJ, 481, 396

de Graauw, T., \& Helmich, F. P. 2001, in Proc. ESA Symp., The Promise of the Herschel Space Observatory, ESA SP-460, 45

Doty, S. D., \& Neufeld, D. A. 1997, ApJ, 489, 122

Doty, S. D., van Dishoeck, E. F., van der Tak, F. F. S., \& Boonman, A. M. S. 2002, A\&A, 389, 446

Doty, S. D., Schöier, F. L., \& van Dishoeck, E. F. 2004, A\&A, 418, 1021

Feigelson, E. D., \& Montmerle, T. 1999, ARA\&A, 37, 363

Forbrich, J., Preibisch, T., \& Menten, K. 2005, Star Formation in the Era of Three Great Observatories

Giannini, T., Nisini, B., \& Lorenzetti, D. 2001, ApJ, 555, 40

Glassgold, A. E., Najita, J., \& Igea, J. 2004, ApJ, 615, 972

Gredel, R., Lepp, S., Dalgarno, A., \& Herbst, E. 1989, ApJ, 347, 289

Hamaguchi, K., Corcoran, M. F., Petre, R., et al. 2005a, ApJ, 623, 291
Hamaguchi, K., Corcoran, M. F., Petre, R., et al. 2005b, Star Formation in the Era of Three Great Observatories

Hogerheijde, M. R., van Dishoeck, E. F., Blake, G. A., \& van Langevelde, H. J. 1997, ApJ, 489, 293

Hollenbach, D., \& McKee, C. F. 1989, ApJ, 342, 306

Imanishi, K., Koyama, K., \& Tsuboi, Y. 2001, ApJ, 557, 747

Jensen, M. J., Bilodeau, R. C., Safvan, C. P., et al. 2000, ApJ, 543, 764

Jørgensen, J. K., Schöier, F. L., \& van Dishoeck, E. F. 2002, A\&A, 389, 908

Jørgensen, J. K. 2004, A\&A, 424, 589

Jørgensen, J. K., Schöier, F. L., \& van Dishoeck, E. F. 2005a, A\&A, 437, 501

Jørgensen, J. K., Lahuis, F., Schöier, F. L., et al. 2005b, ApJ, 631, L77

Jørgensen, J. K., Bourke, T. L., Myers, P. C., et al. 2005c, ApJ, 632, 973

Koyama, K., Hamaguchi, K., Ueno, S., Kobayashi, N., \& Feigelson, E. D. 1996, PASJ, 48, L87

Lee, H.-H., Herbst, E., Pineau des Forets, G., Roueff, E., \& Le Bourlot, J. 1996, A\&A, 311, 690

Looney, L. W., Mundy, L. G., \& Welch, W. J. 2000, ApJ, 529, 477

Maloney, P. R., Hollenbach, D. J., \& Tielens, A. G. G. M. 1996, ApJ, 466, 561

Maret, S., Ceccarelli, C., Caux, E., Tielens, A. G. G. M., \& Castets, A. 2002, A\&A, 395, 573

Maret, S., Ceccarelli, C., Caux, E., et al. 2004, A\&A, 416, 577

McCall, B. J., Geballe, T. R., Hinkle, K. H., \& Oka, T. 1999, ApJ, 522, 338

Meixner, M., \& Tielens, A. G. G. M. 1993, ApJ, 405, 216

Melnick, G. J. 2004, Adv. Space Res., 34, 511

Millar, T. J., Farquhar, P. R. A., \& Willacy, K. 1997, A\&AS, 121, 139

Nisini, B., Giannini, T., \& Lorenzetti, D. 2002, ApJ, 574, 246

Pagani, L., Olofsson, A. O. H., Bergman, P., et al. 2003, A\&A, 402, L77

Preibisch, T., Kim, Y. C., Favata, F., et al. 2005, ApJS, 160, 401

Schöier, F. L., Jørgensen, J. K., van Dishoeck, E. F., \& Blake, G. A. 2002, A\&A, 390,1001

Schöier, F. L., Jørgensen, J. K., van Dishoeck, E. F., \& Blake, G. A. 2004, A\&A, 418,185

Sims, I. et al. 2006, in Astrochemistry across the Universe, IAU Symp., 231, ed. D. C. List, E. Herbst, \& G. A. Blake (Cambridge Univ. Press), in press

Spaans, M., Hogerheijde, M. R., Mundy, L. G., \& van Dishoeck, E. F. 1995, ApJ, 455, L167

Spaans, M., \& van Dishoeck, E. F. 2001, ApJ, 548, L217

Stäuber, P., Doty, S. D., van Dishoeck, E. F., Jørgensen, J. K., \& Benz, A. O. 2004, A\&A, 425, 577

Stäuber, P., Doty, S. D., van Dishoeck, E. F., \& Benz, A. O. 2005, A\&A, 440, 949

Tielens, A. G. G. M., Tokunaga, A. T., Geballe, T. R., \& Baas, F. 1991, ApJ, 381, 181

van der Tak, F. F. S., \& van Dishoeck, E. F. 2000, A\&A, 358, L79

van der Tak, F. F. S., van Dishoeck, E. F., Evans, N. J., \& Blake, G. A. 2000, ApJ, 537, 283

van der Tak, F., Walmsley, M., Herpin, F., \& Ceccarelli, C. 2006, A\&A, 447, 1011

van Dishoeck, E. F., \& Blake, G. A. 1998, ARA\&A, 36, 317 